\title{
A straightforward synthesis of 3-substituted azetidinic amino acids
}

\author{
Mangaleswaran Sivaprakasham, ${ }^{\text {a }}$ François Couty,,${ }^{a, *}$ Gwilherm Evano, ${ }^{a}$ B. Srinivas, ${ }^{\text {b }}$ R. \\ Sridhar, ${ }^{b}$ and K. Rama Rao ${ }^{b}$ \\ a Institut Lavoisier, UMR 8180, Université de Versailles, 45, avenue des Etats-Unis, 78035, \\ Versailles Cedex, France \\ ${ }^{b}$ Organic Chemistry Division-I, Indian Institute of Chemical Technology, Hyderabad-500 007, \\ India \\ E-Mail:couty@chimie.uvsq.fr
}

Dedicated to Professor A. Krief on the occasion of his $65^{\text {th }}$ anniversary

\begin{abstract}
A series of enantiomerically pure $N, N$-disubstituted $\beta$-amino alcohols were chlorinated by treatment with thionyl chloride. This reaction afforded a mixture of regioisomeric $\beta$-amino chlorides that could be equilibrated to the more stable regioisomer by heating in DMF. The secondary chlorides thus obtained were engaged in an intramolecular anionic ring-closure to give access to fully protected enantio- and diastereoisomerically pure 2,3-cis-disubstituted azetidinic amino acid. One of the latter was deprotected for inclusion in a tripeptide sequence. This synthetic methodology was applied for $N, N$-disubstituted $\gamma$-amino alcohols, leading to 3 - or 5substituted proline derivatives but with a low 2,3- or 2,5- diastereoselectivity.
\end{abstract}

Keywords: Azetidines, amino-acids, amino-alcohols

\section{Introduction}

Conformationally constrained amino acids have been the subject of growing interests within past years in peptide chemistry. These modified amino acids can be used as tools for the modification of the secondary structure of peptides of biological relevance allowing a better knowledge of the shape of the catalytic site within the biomolecule and leading ultimately to the development of peptide-derived pharmaceutical drugs. ${ }^{1}$ In this vast area of research, the asymmetric synthesis of novel $\alpha$-amino acids, in which the constraint is brought by an heterocycle that holds the nitrogen is particularly rich, as illustrated by the synthesis of proline derivatives, ${ }^{2}$ as well as pipecolic acid derivatives. ${ }^{3}$ In contrast, the asymmetric synthesis of cyclic four-membered azetidinic amino acids is less documented. Yet, since the discovery by Fowden ${ }^{4}$ nearly fifty years ago (1955) of L- 
Azetidine-2-carboxylic acid 1, from the liliaceae Convallaria Majalis (lily-of-the-valley) (Fig.1), this non proteinogenic amino acid homologue of proline has been the subject report of impressive biological acivities, ${ }^{5}$ and the modification of the structure of proteins brought by the replacement of proline by 1 has been investigated in detail. ${ }^{6}$ In the last decade, the asymmetric synthesis of substituted derivatives of 1 has therefore been the subject of intense investigations. ${ }^{7}$ This article follows a preliminary communication ${ }^{8}$ reporting the asymmetric synthesis of 2,3-cissubstituted derivatives 2 of this amino acid through a stereoselective anionic intramolecular alkylation and there upon the attempts to generalise this synthetic methodology for the preparation of proline derivatives 3 and 4 .
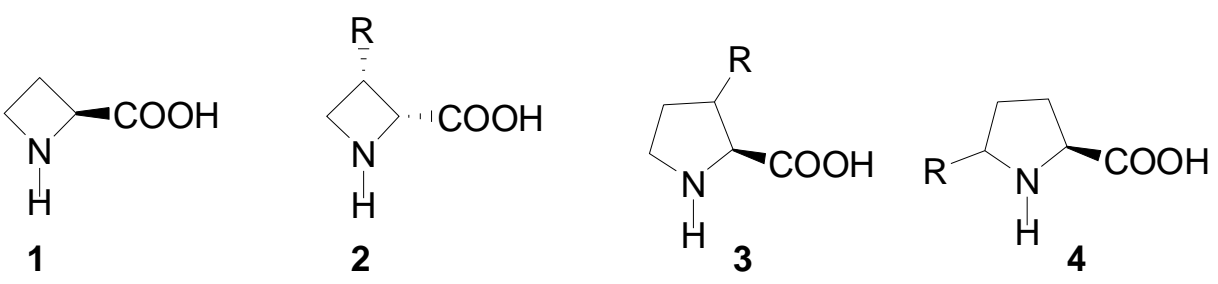

\section{Figure 1}

\section{Results and Discussion}

\section{Synthesis of optically pure $\beta$-amino chlorides}

The synthetic scheme envisioned to access 2 is based on an anionic intramolecular alkylation of amino chloride $\mathbf{6}$, which was prepared from the corresponding $\beta$-amino alcohol $\mathbf{5}$, bearing an electron withdrawing group on the nitrogen atom, which was able to stabilize an adjacent carbanion (Scheme 1).

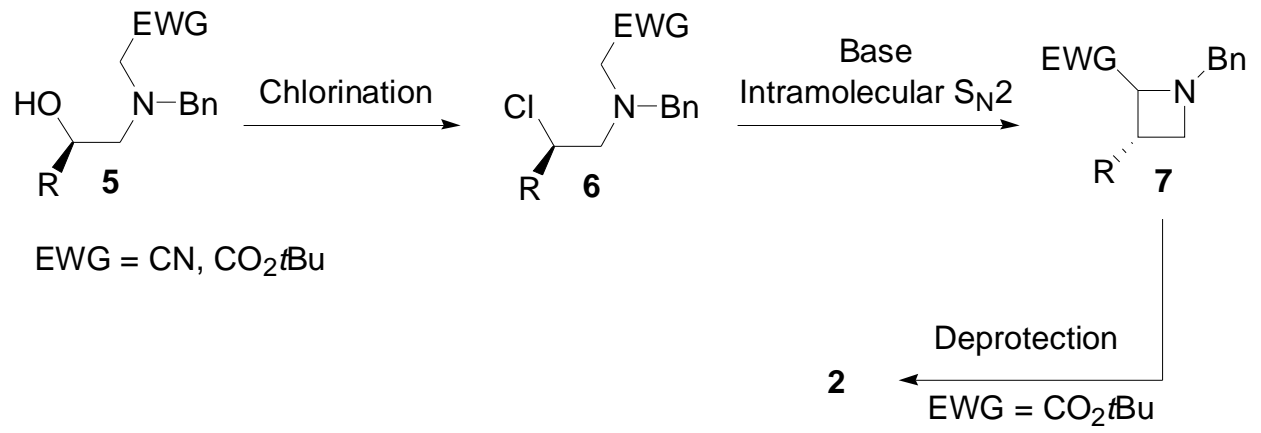

\section{Scheme 1}

This synthetic scheme has been previously validated when $\mathrm{R}=\mathrm{Ph}$ and $\mathrm{EWG}=\mathrm{CN},{ }^{9}$ and the retention of configuration during the chlorination step was explained by a double inversion, involving an intermediate aziridinium ion $\mathbf{8}$ regioselectively opened by the chloride anion at the benzylic position. Due to the straightforwardness of this synthetic scheme, which leads to 
enantiomerically pure functionalized azetidines of high synthetic potential, ${ }^{10}$ it was highly desirable to generalize this sequence when $\mathrm{R}$ is an alkyl group. However, in this case, the chlorination step leads to regioisomeric chlorides $\mathbf{6}$ and $\mathbf{9}$, due to a low selectivity during the opening of the intermediate aziridinium ion by the chloride anion (Scheme 2). We report herein a solution to this problem of regioselectivity and its application to the synthesis of 2 with R being different from an aromatic ring, thus considerably enhancing the generality of this sythetic sequence.

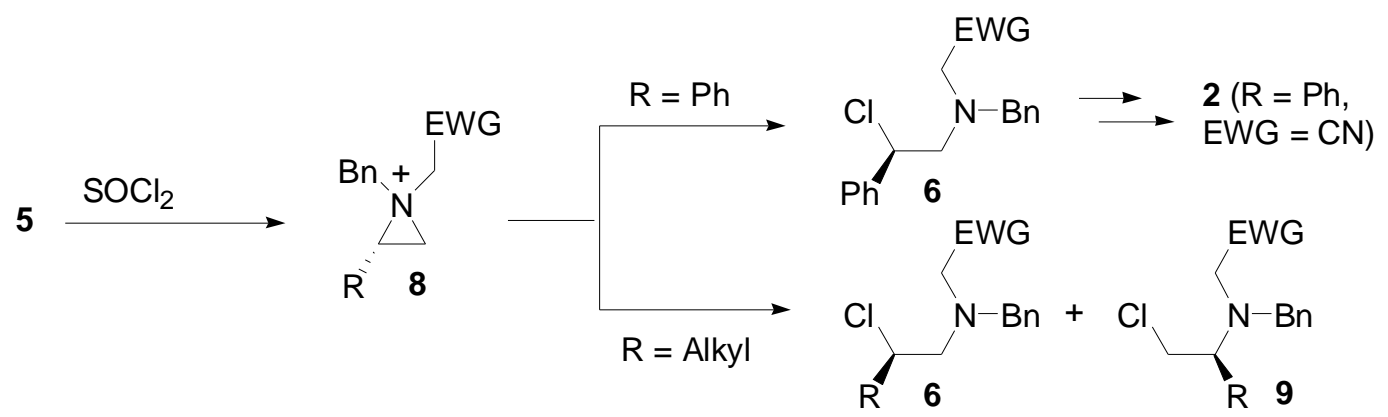

Scheme 2

The $\beta$-amino alcohol substrates 10-20 were prepared in order to study the regioselectivity of the chlorination step (Table 1). These compounds were made either from commercially available enantiomerically pure $\beta$-amino alcohols (for 10-16), from $(S)-O$-benzyl tyrosinol (for 17), ${ }^{11}$ or from $(2 S, 3 R)$ - $O$-benzyl threoninol (for 20), ${ }^{12}$ following a two-step sequence involving $N$ benzylation ( $\mathrm{PhCHO}, 4 \AA$ Molecular sieves, then $\mathrm{NaBH}_{4}$ ) and $N$-alkylation with either bromoacetonitrile (for 10-13: $\mathrm{K}_{2} \mathrm{CO}_{3}, \mathrm{CH}_{3} \mathrm{CN}$ ) or tert-butyl bromoacetate (for 14-20: $\mathrm{NaHCO}_{3}$, NaI, DMF).

Amino alcohols 18 and 19 were prepared from the corresponding epoxides by reaction with either isopropylamine or benzylamine in the presence of $\beta$-cyclodextrin $(\beta-C D)$ following our earlier described methodology. ${ }^{14}$ The resulting amino alcohols were obtained with good yields and high ee's (Scheme 3) and were transformed into 29 and $\mathbf{3 0}$ through the above described alkylation procedure.

\section{Scheme 3}

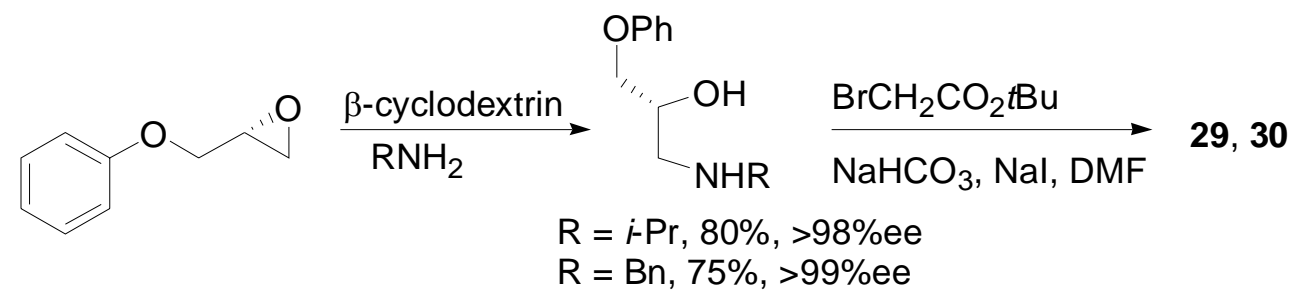

All these $\beta$-amino alcohols were chlorinated by treatment with thionyl chloride in DCM to furnish a regioisomeric mixture of chlorides in good yields. Heating this mixture in DMF at $65^{\circ} \mathrm{C}$ for the time specified in Table 1 resulted in a complete equilibration to give the secondary chlorides 21-31. These conditions were adjusted in order to minimize degradation and the secondary chlorides were obtained in high overall yields and $>98 \%$ regioisomeric purity. 
Table 1. Regioselective chlorination of $\beta$-amino alcohols

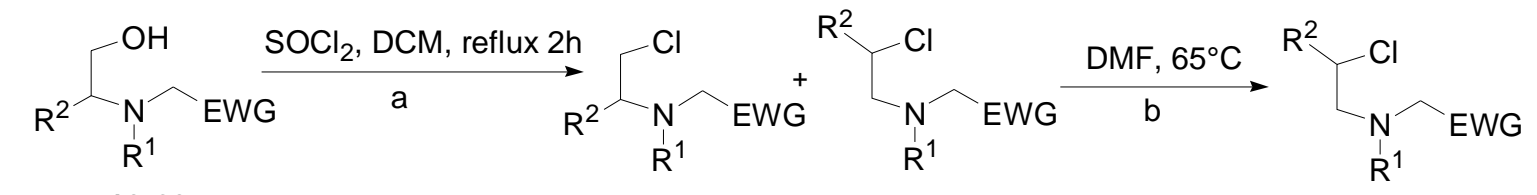

10-20

21-31

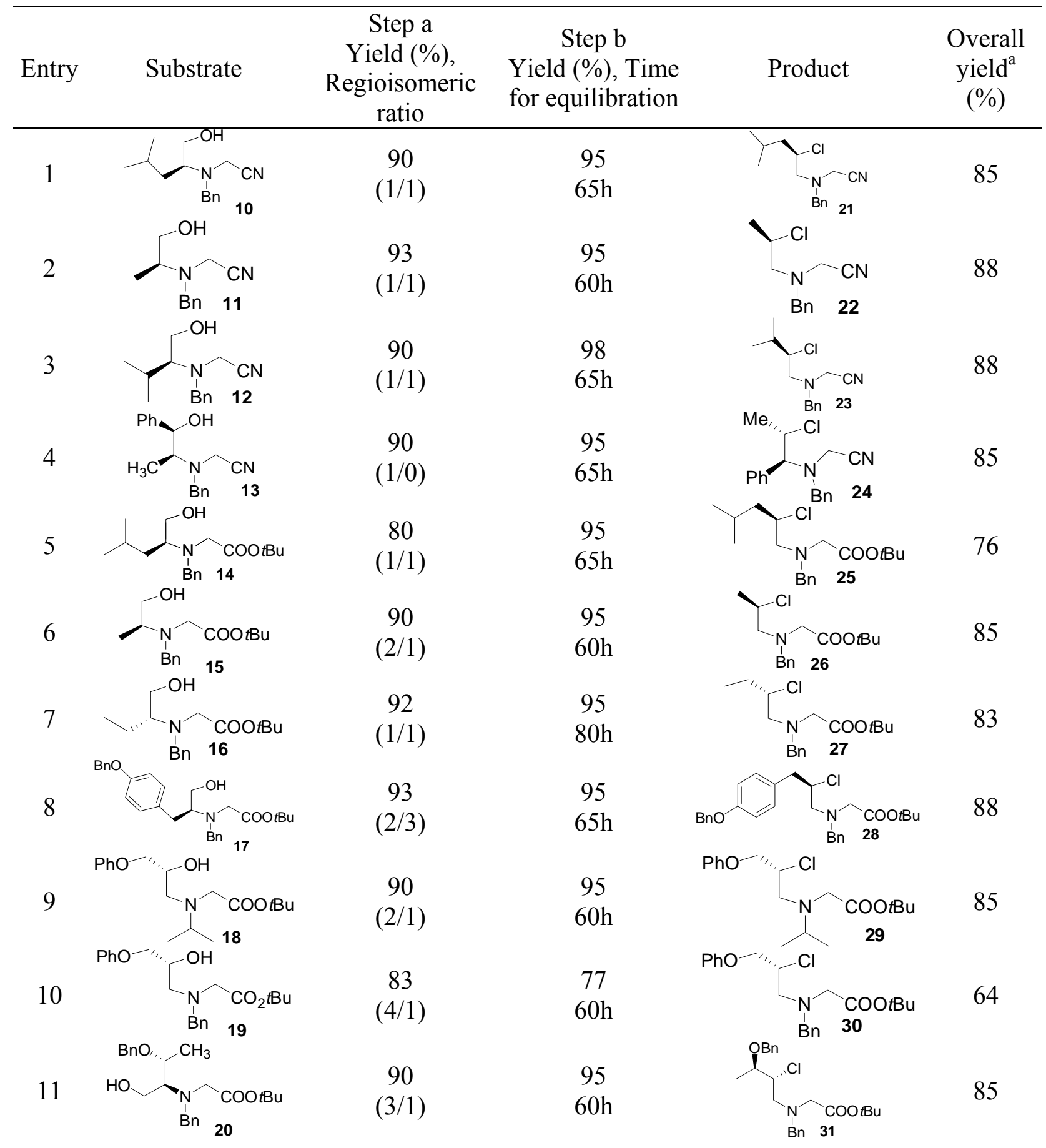

${ }^{\mathrm{a}}$ Yield of pure isolated product. 
The high regioselectivity obtained of the chlorination step can be explained by the occurrence of an equilibration through an intermediate aziridinium ion (Scheme 4). Upon heating in DMF, both primary and secondary chlorides can produce an aziridinium ion through intramolecular alkylation. Chloride anion can then reattack this electrophilic intermediate to give either primary or secondary chloride. The accumulation of the secondary chloride in the reaction mixture can be explained by the fact that it is less prone to give back the aziridinium ion, due to the crowding of the electrophilic center. It should be noted that a very similar behaviour with azetidinium ions has been reported recently. ${ }^{15}$ Long reaction times required for equilibration suggests that the opening at the less hindered position to give back the primary chloride is the more rapid process. Therefore, the primary chloride is the kinetic product, and the secondary chloride is the thermodynamic product and that these compounds are obtained without loss of the optical purity was demonstrated by the high optical purity of the azetidines derived from these products (vide supra). The kinetic opening by a nucleophile at the less hindered position can be easily evidenced: heating a 6/4 mixture of chlorides derived from 11 in $\mathrm{MeOH}$ gave a mixture of primary ether $\mathbf{3 3}$ and unreacted secondary chloride 22; no trace of secondary ether could be detected in the reaction mixture.
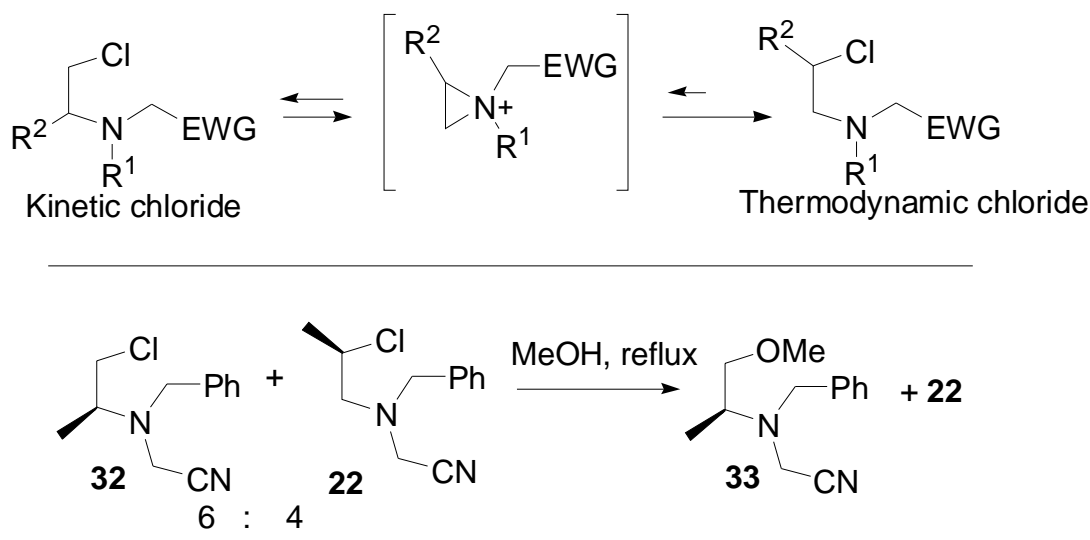

\section{Scheme 4}

The regioselectivity issue being solved, we next focused on the nucleophilic ring closure in order to form the azetidine ring. Therefore, chlorides 21-24 bearing a cyanomethyl moiety on the nitrogen were treated with LiHMDS at $-78^{\circ} \mathrm{C}$, following our previously described conditions. ${ }^{9}$ Cyclization occured in high yield with 21 and 22, but not with 23, in which the electrophilic center is more sterically crowded and decomposition was observed with ephedrin-derived chloride 24. However, the 2,3-diastereoselectivity in the produced azetidines 34-39 was low in all cases, and the diasteroisomers were not easily separable. We therefore switched to the chlorides 25-31 bearing a large tert-butyl ester, in the hope of increasing the diasteroselectivity. As a matter of fact, the enolates derived from these esters proved to be less reactive than their aminonitrile homologues and it was crucial to use HMPA (hexamethylphosphoramide) as an additive (10\% volume with THF) to induce the cyclization with these substrates. Nonetheless, under these conditions, cyclized compounds 40-45 were obtained in good yields and we were pleased to note that it occurred with total 2,3-cis diastereoselectivity. Results of these experiments are summarized in Table 2. 
Table 2. Intramolecular alkylation of chlorides 21-30

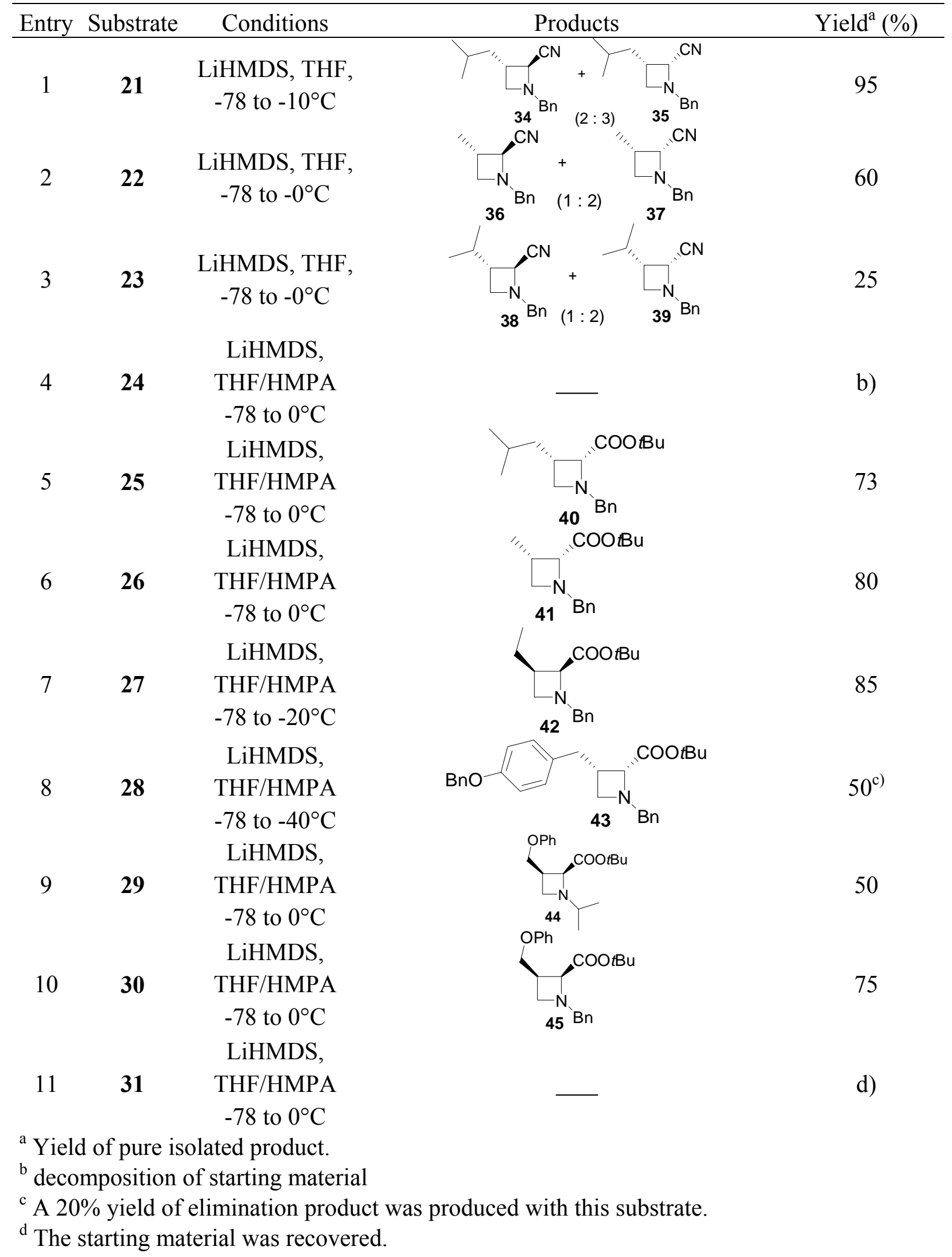


Particularly noteworthy in these experiments is the high 2,3-cis-diastereoselectivity observed in the course of the cyclization. The 2,3-relative configuration in the produced azetidines was determined by NOE experiments performed with 41 and 44 (NOE enhancements are shown in Figure 2).

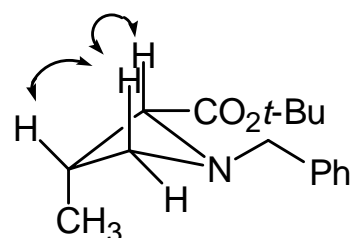

41

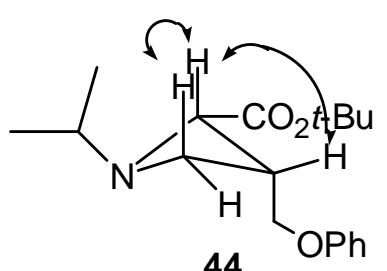

44

Figure 2. NOE enhancements in $\mathbf{4 1}$ and $\mathbf{4 4 .}$

The optical purity of the azetidine $\mathbf{4 1}$ and ent-41 (prepared from ent-15) was determined by NMR using (S)- $\alpha$-(trifluoromethyl)benzyl alcohol as chiral solvent ${ }^{16}$ and was found to be greater than $95 \%$, demonstrating that no loss of the optical purity occurred during the equilibration process.

The rationalization of the observed cis-2,3 diastereoselectivity is not simple. The first question to be answered is whether the reaction is kinetically controlled or if further enolisation of the produced azetidine induces a thermodynamic control. In order to address this point, we have treated azetidine 41 under the conditions of the cyclization (LiHMDS in THF/HMPA) followed by quenching at $0^{\circ} \mathrm{C}$ with methanol- $d_{4}$. Since the starting product was recovered unchanged and did not show any incorporation of deuterium, we could conclude that it is not enolized in the reaction mixture. This observation is however not sufficient to conclude that a kinetic control is operating, since we cannot rule out an enolization of a 2,3-trans azetidine into the observed 2,3-cis isomer. If a kinetic control is assumed, then a clear cut explanation of the selectivity through the examination of the possible transition states is precluded by at least three important questions that we have not been able to answer so far: (i) what is the stereochemistry ( $Z$ or $E$ ) of the reactive enolate? (ii) what is the conformation of the azetidine ring in the transition state? and (iii) can the ester enolate support a pseudo axial position on the azetidine ring in the transition state? All the possibilities brought by these questions suggest at least eight possible transition states that we examined with the aid of molecular model: from this study, a preferred transition state did not emerge. Furthermore, in this first insight into the mechanism, the possible role of HMPA was neglected. It is therefore clear that further experimentation coupled with molecular modeling will be necessary to rationalize this disatereoselectivity. From a more pratical viewpoint, it should be noted that although the stereoselectivity is very good, the ease of this intramolecular alkylation depends on the steric crowding of the electrophilic center: compounds 24 and 31, both bearing a substituent alpha to the reacting center, failed to give any azetidine. Furthemore, compound $\mathbf{2 8}$ gave substantial amount of elimination product, due to a benzylic position alpha to the reacting center. Thus, this series of experiments delineates well the scope of this reaction. 
In order to demonstrate that these azetidinic amino acids are suitable for peptide chemistry, amino ester 41 was included in a tripeptide, as shown in Scheme 5. Thus, cleavage of the $t$ butyl ester in 41 with TFA was followed by coupling with $(L)$-Ala-OMe to give 49. $N$-Debenzylation of 49 was then followed by coupling with $(L)-N$-Boc-Ala, to furnish tripeptide 50 with modest yield. The use of the BOP reagent proved to be necessary for this step to attain a reasonable yield (Scheme 5). This tripeptide was shown to exist as a mixture of two rotamers by ${ }^{1} \mathrm{H}$ NMR. Alternatively, $N$-Benzyl protecting group in $\mathbf{4 1}$ was changed in good yield to a Boc group to give 46. The latter was fully depotected with TFA, to give amino acid 47 after ion-exchange chromatography. This unreported amino acid can be viewed as a conformationally restricted analogue of valine.<smiles>CCOC(=O)[C@H]1C(C)CN1Cc1ccccc1</smiles>

41

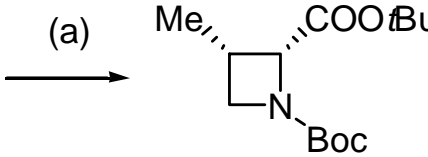

46

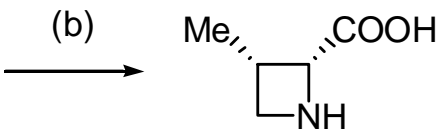

47 (c)<smiles>C[C@@H]1C[NH+](Br)[C@@H]1C(=O)O</smiles>

48<smiles>CC(=O)C(C)NC(=O)[C@@H]1C(C)CN1Cc1ccccc1</smiles>

49

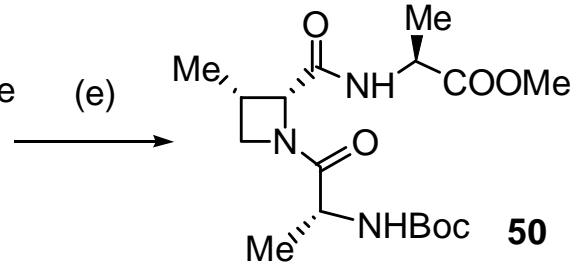

Scheme 5. (a) $\mathrm{H}_{2}$, (Boc) $)_{2} \mathrm{O}, \mathrm{Pd}(\mathrm{OH})_{2}$ cat., EtOH, r.t., $2 \mathrm{~h}(89 \%)$. (b) TFA in DCM, r.t. 12h, then DOWEX (95\%). (c) TFA in DCM, r.t., 12h (95\%). (d) BOP reagent, $(L)$-Ala-OMe, HCl, Et ${ }_{3} \mathrm{~N}$, $\mathrm{MeCN}$, r.t., 16h, (65\%). (e) (i) $\mathrm{H}_{2}, \mathrm{Pd}(\mathrm{OH})_{2}$ cat., $\mathrm{MeOH} / \mathrm{AcOH}$, r.t., 3h, (95\%). (ii) BOP reagent, $N$-Boc- $(L)$-Ala, $\mathrm{Et}_{3} \mathrm{~N}, \mathrm{MeCN}$, r.t., 16h, $(50 \%)$.

After success in synthesizing the enantio- and diasteromerically pure 3-substituted azetidinic amino acids, we planned to extrapolate the same strategy for the synthesis of substituted proline derivatives. In this regard, the desired enantiomerically pure $\gamma$-amino alcohol 56, homologue to $\mathbf{1 5}$ was synthesized from $\mathbf{5 3}^{17}$ through a three steps sequence involving $N$-benzylation, reduction of the ester moiety and alkylation with tert-butylbromoacetate, as depicted in Scheme 6. 

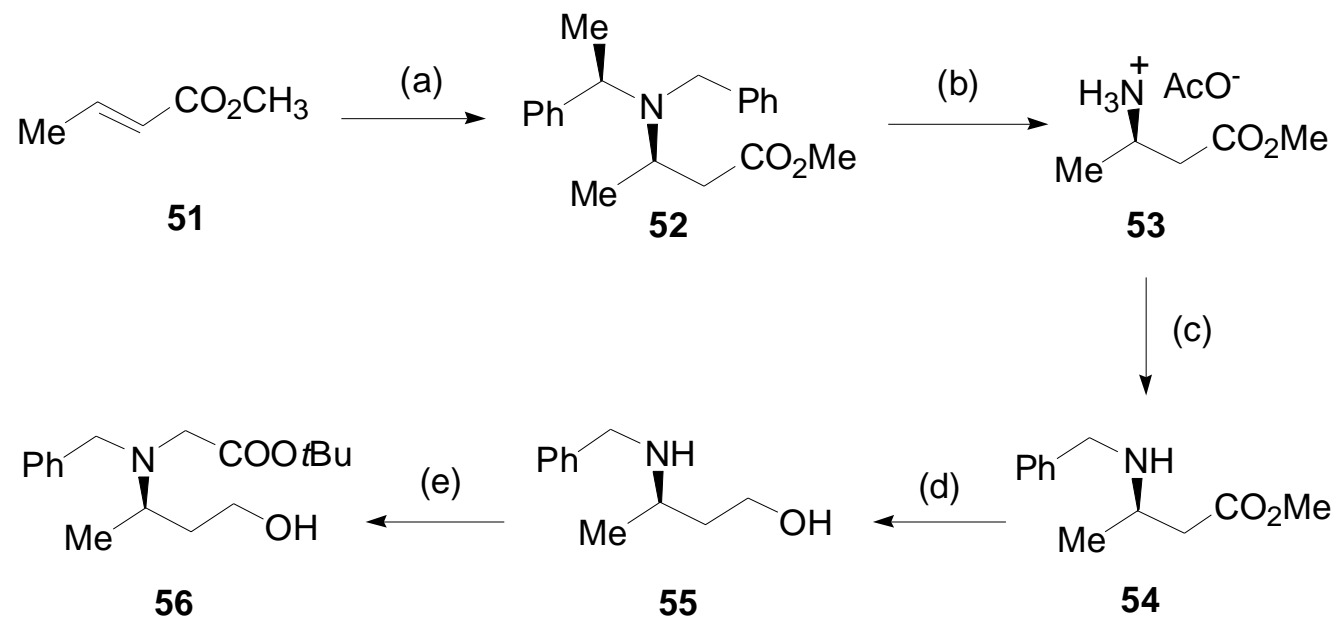

Scheme 6. (a) (R)-Lithium $N$-benzyl- $N$-1-phenylethylamide, $-78^{\circ} \mathrm{C}, 15 \mathrm{mn},(80 \%)$. (b) $\mathrm{H}_{2}$, $\mathrm{Pd}(\mathrm{OH})_{2}$ cat., $\mathrm{MeOH} / \mathrm{AcOH} / \mathrm{H}_{2} \mathrm{O}$, (84\%). (c) (i) Benzaldehyde, $4 \AA \mathrm{MS}, \mathrm{DCM}$, 3h. (ii) $\mathrm{NaBH}_{4}$, EtOH, r.t., 12h, (60\% overall). (d) $\mathrm{LiAlH}_{4}$, THF, reflux, 12h, (97\%). (e) tert-Butylbromoacetate, $\mathrm{NaHCO}_{3}$, NaI, DMF, (57\%).

The synthesized chiral amino alcohol $\mathbf{5 6}$ was then transformed into the primary chloride $\mathbf{5 7}$ which was subjected to cyclization under set conditions and the reaction gave the desired proline derivatives $\mathbf{5 8}$ and $\mathbf{5 9}$ with very good yield but with poor diasteroselectivity (Scheme 7). The relative stereochemistries in these proline derivatives was assigned by comparison of the ${ }^{1} \mathrm{H}$ NMR spectra with the closely related cis and trans 5-heptyl analogues. ${ }^{18}$ It is interesting to note here that the chlorination step leading to $\mathbf{5 7}$ occurred with high regioselectivity, suggesting that equilibration through an intermediate azetidinium ions is not operative under these conditions.

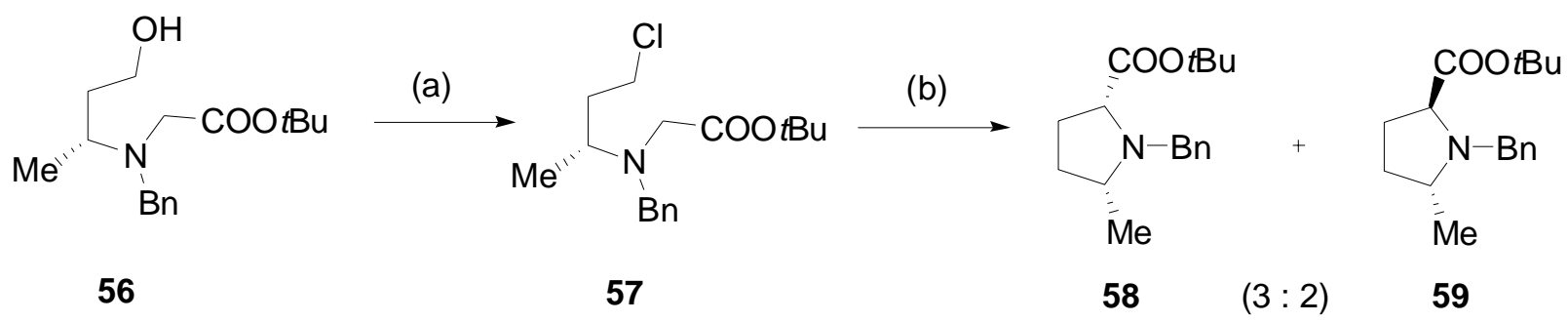

Scheme 7. (a) $\mathrm{SOCl}_{2}$, DCM, reflux, 1.5h, (78\%). (b) LiHMDS, THF/HMPA, -90 to $0^{\circ} \mathrm{C}$, (98\%).

Having failed to get a good diasteroselectivity for the synthesis of 5-substituted proline derivatives we then focussed our attention towards 3-subsituted proline derivatives. Starting from commercially available racemic alcohol 60, a similar sequence of $N$-benzylation and $N$ alkylation gave $\mathbf{6 1}$ which was chlorinated regioselectively to give the chloride 62. Finally, this compound, subjected to intramolecular cyclization using LiHMDS, furnished mixture of diasteroisomers 63 with high yield but poor diasteroselectivity (Scheme 8). 


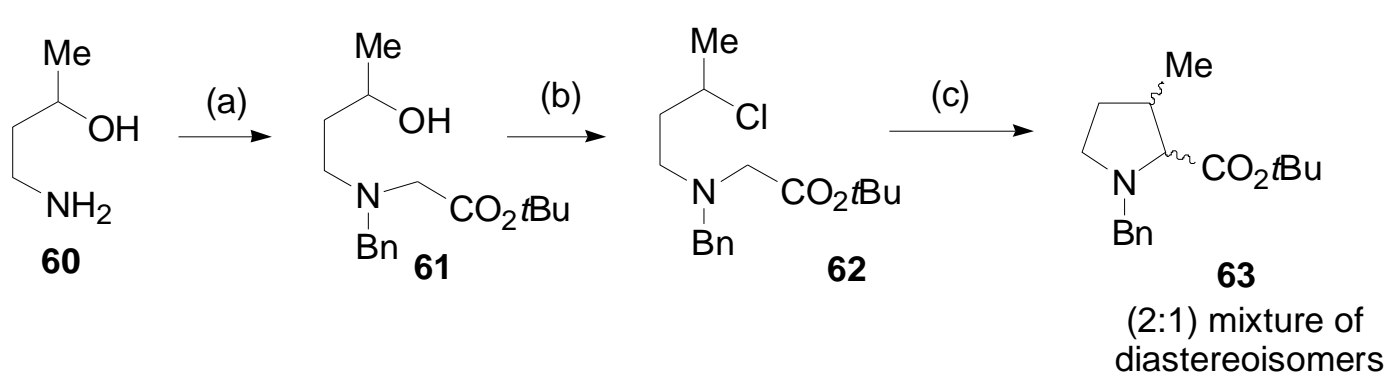

Scheme 8. (a) (i) Benzaldehyde, $4 \AA \mathrm{MS}, \mathrm{DCM}$, 3h. (ii) $\mathrm{NaBH}_{4}$, EtOH, r. t., 12h, (iii) tertButylbromoacetate, $\mathrm{NaHCO}_{3}$, NaI, DMF, (63\% overall). (b) $\mathrm{SOCl}_{2}, \mathrm{DCM}$, reflux, 1.5h, (93\%). (c) LiHMDS, THF/HMPA, -90 to $0^{\circ} \mathrm{C},(98 \%)$.

In conclusion, we have shown that diastereo- and enantiomerically pure cis-2,3-substituted azetidines can be prepared in a straighforward way from $\beta$-amino alcohols. We have also demonstrated that such azetidinic amino acids can be fully deprotected or included using conventional chemistry in a peptidic sequence. Finally, the extension of this synthetic sequence to synthesize proline derivatives has been studied and though the yield was very good, the crucial cyclization step occured with a low diasteroselectivity. Applications of these azetidinic amino acids will be reported in due course of time.

\section{Acknowledgements}

IFCPAR (Indo-French Centre for the promotion of Advanced Research) is gratefully acknowledged for financial support.

\section{Experimental Section}

General Procedures. ${ }^{1} \mathrm{H}$ and ${ }^{13} \mathrm{C}$ spectra $\left(\mathrm{CDCl}_{3}\right.$ solution unless otherwise stated) were recorded on a Brüker AC 200 or 300 spectrometer at $200,300\left({ }^{1} \mathrm{H}\right), 50.3$ and $75.5\left({ }^{13} \mathrm{C}\right) \mathrm{MHz}$; chemical shifts are reported in ppm from TMS. Optical rotations were determined with a Perkin Elmer 141 instrument. All reactions were carried out under argon. Column chromatography was performed on silica gel 230-400 mesh by using various mixtures of petroleum ether (PE), ethyl acetate $(\mathrm{AcOEt})$ and diethyl ether $\left(\mathrm{Et}_{2} \mathrm{O}\right)$. TLCs were run on Merck Kieselgel $60 \mathrm{~F}_{254}$. Melting points were uncorrected. THF was distilled from sodium/benzophenone ketyl. Dichloromethane was distilled from calcium hydride. The mention of "usual workup" means: (i) decantation of the organic layer; (ii) extraction of the aqueous layer with ether; (iii) washing the combined organic layers with brine and drying of the combined organic phases over $\mathrm{MgSO}_{4}$ and (iv) solvent evaporation under reduced pressure. Compositions of stereoisomeric mixtures were determined by NMR analysis on crude products before any purification. Mass spectra were recorded on a Hewlett-Packard MS Engin HP5989B equipped with an ESI source Analytica Branford. HRMS 
spectra were performed by the "Service de spectrometrie de masse" in Strasbourg University. Elementary analyses were performed by the "Service de Microanalyses" in Gif sur Yvette.

\section{General procedure for the preparation of chlorides (GP-1)}

A solution of amino alcohol $(1 \mathrm{mmol})$ in DCM $(5 \mathrm{~mL})$ was cooled to $0^{\circ} \mathrm{C}$ under argon. Thionyl chloride $(2.05 \mathrm{mmol})$ was added dropwise and the resulting mixture was refluxed for $2 \mathrm{~h}$. After the completion of the reaction, the reaction mixture was cooled to room temperature and quenched by addition of saturated aqueous solution of $\mathrm{NaHCO}_{3}(10 \mathrm{~mL})$. The aqueous layer was extracted with diethylether $(2 \times 15 \mathrm{~mL})$ followed by usual workup gave a mixture of chlorides that was purified by flash chromatography. The formed chlorides $(1 \mathrm{mmol})$ were then dissolved in DMF $(10 \mathrm{~mL})$ and heated at $60{ }^{\circ} \mathrm{C}$ for the time specified in Table 1. After completion of the reaction, DMF was removed under vacuo and the crude residue was purified by flash chromatography.

(R)-(-)-[N-(2-Chloro-4-methyl-pentyl)-benzylamino]acetonitrile 21. (Table 1, Entry 1) Prepared according to GP-1. The pure compound was obtained after flash chromatography $\left(\mathrm{SiO}_{2}\right.$, PE/AcOEt, 9:1) as a colourless oil. Yield: $1.94 \mathrm{~g}(85 \%) ; R_{\mathrm{f}}=0.82$ (PE/AcOEt, 9:1); $[\alpha]_{\mathrm{D}}{ }^{20}:-2.0$ $\left(\mathrm{c}=0.80, \mathrm{CHCl}_{3}\right) .{ }^{1} \mathrm{H} \mathrm{NMR}\left(200 \mathrm{MHz}, \mathrm{CDCl}_{3}, 25{ }^{\circ} \mathrm{C}\right): \delta=0.91\left(\mathrm{~d}, J=6.6 \mathrm{~Hz}, 3 \mathrm{H}, \mathrm{CH}_{3}\right) 0.95$ (d, $\left.J=6.6 \mathrm{~Hz}, 3 \mathrm{H}, \mathrm{CH}_{3}\right), 1.50-1.69\left(\mathrm{~m}, 2 \mathrm{H}, \mathrm{CH}_{2} \mathrm{CH}\left(\mathrm{CH}_{3}\right)_{2}\right), 1.82-2.20\left(\mathrm{~m}, 1 \mathrm{H}, \mathrm{CH}\left(\mathrm{CH}_{3}\right)_{2}\right), 2.90$ (dd, $\left.J=7.5 \mathrm{~Hz}, J=2.7 \mathrm{~Hz}, 2 \mathrm{H}, \mathrm{NCH}_{2} \mathrm{CHCl}\right) 3.53\left(\mathrm{~s}, 2 \mathrm{H}, \mathrm{NCH}_{2} \mathrm{CN}\right.$ ), 3.76 (s, $2 \mathrm{H}, \mathrm{NCH}_{2} \mathrm{Ph}$ ), 3.81-4.10 (m, $\left.1 \mathrm{H}, \mathrm{NCH}_{2} \mathrm{CHCl}\right), 7.28-7.48(\mathrm{~m}, 5 \mathrm{H}, \mathrm{Ph} H) \mathrm{ppm} .{ }^{13} \mathrm{C} \mathrm{NMR}\left(75 \mathrm{MHz}, \mathrm{CDCl}_{3}, 25\right.$ $\left.{ }^{\circ} \mathrm{C}\right): \delta=21.3\left(\mathrm{CH}_{3}\right), 23.2\left(\mathrm{CH}_{3}\right), 25.1\left(\mathrm{CH}\left(\mathrm{CH}_{3}\right)_{2}\right), 42.3\left(\mathrm{NCH}_{2} \mathrm{CN}\right), 45.1\left(\mathrm{CH}_{2} \mathrm{CH}\left(\mathrm{CH}_{3}\right)_{2}\right), 58.6$ $\left(\mathrm{NCH}_{2} \mathrm{CHCl}\right), 58.8\left(\mathrm{NCH}_{2} \mathrm{Ph}\right), 61.2\left(\mathrm{NCH}_{2} \mathrm{CHCl}\right), 115.0(\mathrm{CN}), 128.0,128.2,128.5,128.7,129.0$ (CPh) 136.8 (ipso-CPh) ppm. CIMS: $m / z(\%)=265(100)\left[\mathrm{MH}^{+}\right], 238$ (10), 159 (70), $158(23)$, 108 (16), 91 (78). HRMS (ESI) calcd. for $\mathrm{C}_{15} \mathrm{H}_{22} \mathrm{ClN}_{2}\left[\mathrm{MH}^{+}\right]$: 265.1472; found 265.1466.

(R)-(-)-[N-(2-Chloro-propyl)-benzylamino]acetonitrile 22. (Table 1 , Entry 2) Prepared according to GP-1. The pure compound was obtained after flash chromatography $\left(\mathrm{SiO}_{2}\right.$, PE/AcOEt, 9:1) as a colourless oil. Yield: $1.63 \mathrm{~g}(88 \%) ; R_{\mathrm{f}}=0.75$ (PE/AcOEt, 9:1); $[\alpha]_{\mathrm{D}}{ }^{20}:-$ $79.8\left(\mathrm{c}=1.30, \mathrm{CHCl}_{3}\right) .{ }^{1} \mathrm{H} \mathrm{NMR}\left(200 \mathrm{MHz}, \mathrm{CDCl}_{3}, 25^{\circ} \mathrm{C}\right): \delta=1.54\left(\mathrm{~d}, J=6.6 \mathrm{~Hz}, 3 \mathrm{H}, \mathrm{CH}_{3}\right)$, 2.89 (dd, $\left.J=6.9 \mathrm{~Hz}, J=3.1 \mathrm{~Hz}, 2 \mathrm{H}, \mathrm{NCH}_{2} \mathrm{CHCl}\right) 3.52\left(\mathrm{~s}, 2 \mathrm{H}, \mathrm{NCH}_{2} \mathrm{CN}\right), 3.76(\mathrm{~s}, 2 \mathrm{H}$, $\left.\mathrm{NCH}_{2} \mathrm{Ph}\right), 4.03-4.10\left(\mathrm{~m}, 1 \mathrm{H}, \mathrm{CH}_{3} \mathrm{CHCl}\right), 7.22-7.32(\mathrm{~m}, 5 \mathrm{H}, \mathrm{PhH}) \mathrm{ppm} .{ }^{13} \mathrm{C}$ NMR $(50 \mathrm{MHz}$, $\left.\mathrm{CDCl}_{3}, 25{ }^{\circ} \mathrm{C}\right): \delta=23.0\left(\mathrm{CH}_{3}\right), 42.1\left(\mathrm{NCH}_{2} \mathrm{CN}\right), 54.9\left(\mathrm{NCH}_{2} \mathrm{CHCl}\right), 58.7\left(\mathrm{NCH}_{2} \mathrm{Ph}\right), 61.9$ $\left(\mathrm{NCH}_{2} \mathrm{CHCl}\right), 114.9(\mathrm{CN}), 128.0,128.7,129.0(\mathrm{CPh}), 136.7$ (ipso-CPh) ppm. CIMS: $m / z(\%)=$ 225 (31), 223 (100) $\left[\mathrm{MH}^{+}\right], 196$ (14), 159 (32), 91 (31). HRMS (ESI) calcd. for $\mathrm{C}_{12} \mathrm{H}_{16} \mathrm{ClN}_{2}$ $\left[\mathrm{MH}^{+}\right]: 223.1002$; found 223.1012 .

(R)-(-)-[(N-(2-Chloro-3methylbutyl)-benzylamino]acetonitrile 23. (Table 1, Entry 3) Prepared according to GP-1. The pure compound was obtained after flash chromatography $\left(\mathrm{SiO}_{2}\right.$, PE/AcOEt, 9:1) as a colourless oil. Yield: $1.46 \mathrm{~g}(88 \%) ; R_{\mathrm{f}}=0.75$ (PE/AcOEt, 9:1); $[\alpha]_{\mathrm{D}}{ }^{20}:-$ $92.2\left(\mathrm{c}=0.70, \mathrm{CHCl}_{3}\right) .{ }^{1} \mathrm{H} \mathrm{NMR}\left(300 \mathrm{MHz}, \mathrm{CDCl}_{3}, 25^{\circ} \mathrm{C}\right): \delta=0.95\left(\mathrm{~d}, J=6.6 \mathrm{~Hz}, 3 \mathrm{H}, \mathrm{CH}_{3}\right)$, $1.07\left(\mathrm{~d}, J=6.8 \mathrm{~Hz}, 3 \mathrm{H}, \mathrm{CH}_{3}\right), 2.07-2.16\left(\mathrm{~m}, 1 \mathrm{H}, \mathrm{CH}\left(\mathrm{CH}_{3}\right)_{2}\right), 2.90(\mathrm{dd}, J=6.8 \mathrm{~Hz}, J=3.2 \mathrm{~Hz}$, 
$\left.2 \mathrm{H}, \mathrm{NCH}_{2} \mathrm{CHCl}\right), 3.50$ (s, 2H, $\left.\mathrm{NCH}_{2} \mathrm{CN}\right), 3.75$ (s, 2H, $\left.\mathrm{NCH}_{2} \mathrm{Ph}\right), 3.94-4.00(\mathrm{~m}, 1 \mathrm{H}$, $\left.\mathrm{NCH}_{2} \mathrm{CHCl}\right), 7.22-7.45(\mathrm{~m}, 5 \mathrm{H}, \mathrm{Ph} H)$ ppm. ${ }^{13} \mathrm{C} \mathrm{NMR}\left(75 \mathrm{MHz}, \mathrm{CDCl}_{3}, 25{ }^{\circ} \mathrm{C}\right): \delta=16.5\left(\mathrm{CH}_{3}\right)$, $20.3\left(\mathrm{CH}_{3}\right), 31.9\left(\mathrm{CH}\left(\mathrm{CH}_{3}\right)_{2}\right) 42.0\left(\mathrm{NCH}_{2} \mathrm{CN}\right), 58.7\left(\mathrm{NCH}_{2} \mathrm{Ph}\right), 66.5\left(\mathrm{NCH}_{2} \mathrm{CHCl}\right), 114.9(\mathrm{CN})$, 128.0, 128.5, 128.7, 128.8, 129.0 (CPh),136.8 (ipso-CPh) ppm. CIMS: $m / z(\%)=251$ (22) $\left[\mathrm{MH}^{+}\right], 224$ (5), 159 (68), 91 (100). HRMS (ESI) calcd. for $\mathrm{C}_{14} \mathrm{H}_{20} \mathrm{ClN}_{2}\left[\mathrm{MH}^{+}\right]$: 251.1315; found 251.1310 .

(1S,2S)-(+)-(N-(2-Chloro-1-phenylpropyl)-methylamino)acetonitrile 24. (Table 1, Entry 4) Prepared according to GP-1. The pure compound was obtained after flash chromatography $\left(\mathrm{SiO}_{2}\right.$, PE/AcOEt, 9:1) as colourless oil. Yield: $1.43 \mathrm{~g}(85 \%) ; R_{\mathrm{f}}=0.85$ (PE/AcOEt, 9:1); $[\alpha]_{\mathrm{D}}{ }^{20}:+$ $370.0\left(\mathrm{c}=0.60, \mathrm{CHCl}_{3}\right) .{ }^{1} \mathrm{H} \mathrm{NMR}\left(300 \mathrm{MHz}, \mathrm{CDCl}_{3}, 25{ }^{\circ} \mathrm{C}\right): \delta=1.27(\mathrm{~d}, J=6.7 \mathrm{~Hz}, 3 \mathrm{H}$, $\left.\mathrm{CH}_{3} \mathrm{CHCl}\right), 2.48$ (s, 3H, $\left.\mathrm{NCH}_{3}\right), 3.46$ (d, $\left.J=17.3 \mathrm{~Hz}, 1 \mathrm{H}, \mathrm{NCHHCN}\right), 3.47$ (d, $J=8.0 \mathrm{~Hz}, 1 \mathrm{H}$, $\mathrm{PhCHN}), 3.63(\mathrm{~d}, J=17.3 \mathrm{~Hz}, 1 \mathrm{H}, \mathrm{NCH} H C \mathrm{~N}), 4.42-4.62\left(\mathrm{~m}, 1 \mathrm{H}, \mathrm{CH}_{3} \mathrm{CHCl}\right), 7.28-7.45$ (m, $5 \mathrm{H}, \mathrm{Ph} H)$ ppm. ${ }^{13} \mathrm{C} \mathrm{NMR}\left(75 \mathrm{MHz}, \mathrm{CDCl}_{3}, 25{ }^{\circ} \mathrm{C}\right): \delta=22.8\left(\mathrm{CH}_{3}\right), 40.1\left(\mathrm{NCH}_{3}\right), 44.1$ $\left(\mathrm{NCH}_{2} \mathrm{CN}\right), 56.6\left(\mathrm{CH}_{3} \mathrm{CHCl}\right), 72.0(\mathrm{PhCHN}), 114.6(\mathrm{CN}), 128.3,128.7,129.7(\mathrm{CPh}), 135.2$ (ipso-CPh) ppm.; EIMS: $m / z(\%)=196(16)(\mathrm{M}-\mathrm{HCN})^{+}, 187$ (100), 153 (28), 117(23). HRMS (ESI) calcd. for $\mathrm{C}_{12} \mathrm{H}_{16} \mathrm{ClN}_{2}\left[\mathrm{MH}^{+}\right]$: calcd. 223.0997; found 223.0996.

(R)-(-)-[N-(2-Chloro-4-methyl-pentyl)-benzylamino] Acetic acid tert-butyl ester 25. (Table 1, Entry 5) Prepared according to GP-1. The pure compound was obtained after flash chromatography $\left(\mathrm{SiO}_{2}, \mathrm{PE} / \mathrm{AcOEt}, 9: 1\right)$ as colourless oil. Yield: $4.15 \mathrm{~g}(76 \%) ; R_{\mathrm{f}}=0.82$ (PE/AcOEt, 9:1); $[\alpha]_{365}{ }^{20}:-25.4\left(\mathrm{c}=0.90, \mathrm{CHCl}_{3}\right) .{ }^{1} \mathrm{H} \mathrm{NMR}\left(200 \mathrm{MHz}, \mathrm{CDCl}_{3}, 25{ }^{\circ} \mathrm{C}\right): \delta=$ $0.88\left(\mathrm{~d}, J=6.4 \mathrm{~Hz}, 3 \mathrm{H}, \mathrm{CH}_{3} \mathrm{CH}\left(\mathrm{CH}_{3}\right)\right), 0.94\left(\mathrm{~d}, J=6.8 \mathrm{~Hz}, 3 \mathrm{H}, \mathrm{CH}_{3} \mathrm{CH}\left(\mathrm{CH}_{3}\right), 1.50\right.$ (s, 9H, $\left.\mathrm{C}\left(\mathrm{CH}_{3}\right)_{3}\right), 1.51-1.71\left(\mathrm{~m}, 2 \mathrm{H}, \mathrm{CH}_{2} \mathrm{CHCl}\right), 1.85-2.00\left(\mathrm{~m}, 1 \mathrm{H}, \mathrm{CH}\left(\mathrm{CH}_{3}\right)_{2}\right), 3.00(\mathrm{dd}, J=15.0 \mathrm{~Hz}, J$ $=6.7 \mathrm{~Hz}, 1 \mathrm{H}, \mathrm{NCHHCHCl}), 3.06(\mathrm{dd}, J=15.0 \mathrm{~Hz}, J=6.7 \mathrm{~Hz}, 1 \mathrm{H}, \mathrm{NCH} H C H C l), 3.33(\mathrm{~s}, 2 \mathrm{H}$, $\left.\mathrm{NCH}_{2} \mathrm{COO}^{t} \mathrm{Bu}\right), 3.86-4.08\left(\mathrm{~m}, 3 \mathrm{H}, \mathrm{NCH}_{2} \mathrm{Ph}, \mathrm{NCH}_{2} \mathrm{CHCl}\right), 7.27-7.30(\mathrm{~m}, 5 \mathrm{H}, \mathrm{PhH}) \mathrm{ppm} .{ }^{13} \mathrm{C}$

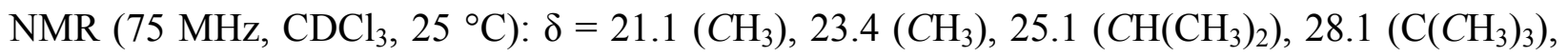
$45.1 \quad\left(\mathrm{CHCH}_{2} \mathrm{CHCl}\right), \quad 55.9 \quad\left(\mathrm{NCH}_{2} \mathrm{COO}^{t} \mathrm{Bu}\right), \quad 58.7 \quad\left(\mathrm{NCH}_{2} \mathrm{Ph}\right), \quad 59.9 \quad\left(\mathrm{NCH}_{2} \mathrm{CHCl}\right), 61.8$ $\left(\mathrm{NCH}_{2} \mathrm{CHCl}\right), 81.0\left(\mathrm{COOC}\left(\mathrm{CH}_{3}\right)_{3}\right), 127.2,128.3,128.9(\mathrm{CPh}), 139.1$ (ipso-CPh), $170.8(\mathrm{CO})$ ppm. EIMS: $m / z(\%)=340(28)\left[\mathrm{MH}^{+}\right], 284$ (20), 238 (100), 234 (41), 178 (32), 91 (85). HRMS (ESI) calcd. for $\mathrm{C}_{19} \mathrm{H}_{31} \mathrm{ClNO}_{2}\left[\mathrm{MH}^{+}\right]$: calcd. 340.2038; found 340.2045.

(R)-(-)-[N-(2-Chloro-propyl)-benzylamino]-acetic acid tert-butyl ester 26. (Table 1, Entry 6) Prepared according to GP-1. The pure compound was obtained after flash chromatography $\left(\mathrm{SiO}_{2}\right.$, PE/AcOEt, 9:1) as colourless oil. Yield: $1.09 \mathrm{~g}(85 \%) ; R_{\mathrm{f}}=0.85$ (Petroleum ether/AcOEt, 9:1); $[\alpha]_{\mathrm{D}}{ }^{20}:-6.6\left(\mathrm{c}=0.70, \mathrm{CHCl}_{3}\right) .{ }^{1} \mathrm{H} \mathrm{NMR}\left(300 \mathrm{MHz}, \mathrm{CDCl}_{3}, 25^{\circ} \mathrm{C}\right): \delta=1.37\left(\mathrm{~s}, 12 \mathrm{H}, \mathrm{C}\left(\mathrm{CH}_{3}\right)_{3}\right.$, $\mathrm{CH}_{3} \mathrm{CHCl}$ ), 2.80 (dd, $\left.J=13.7 \mathrm{~Hz}, J=6.8 \mathrm{~Hz}, 1 \mathrm{H}, \mathrm{NCHHCHCl}\right), 3.19$ (s, 2H, $\mathrm{NCH}_{2} \mathrm{COOtBu}$ ), $3.75(\mathrm{~d}, J=16.8 \mathrm{~Hz}, 1 \mathrm{H}, \mathrm{A}$ of AB system, $\mathrm{NCHHPh}), 3.81$ (d, $J=16.8 \mathrm{~Hz}, 1 \mathrm{H}, \mathrm{B}$ of AB system, $\mathrm{NCH} H \mathrm{Ph}$ ), 3.90 (sextet, $\left.J=6.8 \mathrm{~Hz}, 1 \mathrm{H}, \mathrm{CH}_{3} \mathrm{CH}\left(\mathrm{CH}_{2}\right) \mathrm{Cl}\right), 7.14-7.20(\mathrm{~m}, 5 \mathrm{H}, \mathrm{PhH}) \mathrm{ppm} .{ }^{13} \mathrm{C}$

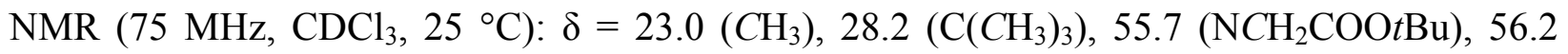

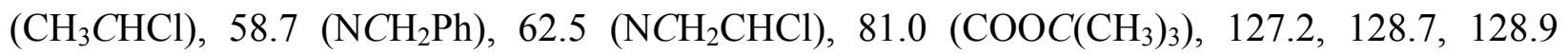
(CPh), 136.8 (ipso-CPh), 170.7 (CO) ppm. EIMS: $m / z(\%)=320$ (27) [M+K $\left.{ }^{+}\right], 264$ (8), 242 (61). HRMS (ESI) calcd. for $\mathrm{C}_{16} \mathrm{H}_{25} \mathrm{ClNO}_{2}\left[\mathrm{MH}^{+}\right]$: 298.1568; found 298.1590. 
(S)-(+)-[N-(2-Chloro-butyl)-benzylamino]-acetic acid tert-butyl ester 27. (Table 1, Entry 7) Prepared according to GP-1. The pure compound was obtained after flash chromatography $\left(\mathrm{SiO}_{2}\right.$, PE/AcOEt, 9:1) as colourless oil. Yield: $1.47 \mathrm{~g}(83 \%) ; R_{\mathrm{f}}=0.85(\mathrm{PE} / \mathrm{AcOEt}, 9: 1) ;[\alpha]_{\mathrm{D}}{ }^{20}:+2.8$ $\left(\mathrm{c}=1.00, \mathrm{CHCl}_{3}\right) .{ }^{1} \mathrm{H} \mathrm{NMR}\left(200 \mathrm{MHz}, \mathrm{CDCl}_{3}, 25^{\circ} \mathrm{C}\right): \delta=1.06\left(\mathrm{t}, J=7.3 \mathrm{~Hz}, 3 \mathrm{H}, \mathrm{CH}_{3}\right), 1.53(\mathrm{~s}$, $\left.9 \mathrm{H}, \mathrm{C}\left(\mathrm{CH}_{3}\right)_{3}\right), 1.60-1.74\left(\mathrm{~m}, 1 \mathrm{H}, \mathrm{CHHCH}_{3}\right), 1.95-2.10\left(\mathrm{~m}, 1 \mathrm{H}, \mathrm{CH}_{\mathrm{CCH}}\right), 3.05(\mathrm{dd}, J=14.1 \mathrm{~Hz}$, $J=6.9 \mathrm{~Hz}, 1 \mathrm{H}, \mathrm{NCHHCHCl}), 3.08(\mathrm{dd}, J=14.1 \mathrm{~Hz}, J=6.9 \mathrm{~Hz}, 1 \mathrm{H}, \mathrm{NCH} H \mathrm{CHCl}), 3.35$ (s, 2H, $\mathrm{NCH}_{2} \mathrm{COOtBu}$ ), 3.85-4.03 (m, 3H, $\left.\mathrm{NCH}_{2} \mathrm{Ph}, \mathrm{CH}_{2} \mathrm{CHCl}\right), 7.24-7.51(\mathrm{~m}, 5 \mathrm{H}, \mathrm{PhH}) \mathrm{ppm} .{ }^{13} \mathrm{C}$

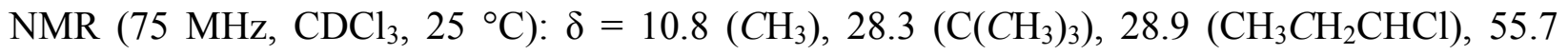
$\left(\mathrm{NCH}_{2} \mathrm{COO}^{t} \mathrm{Bu}\right), \quad 58.7 \quad\left(\mathrm{NCH}_{2} \mathrm{Ph}\right), \quad 60.9 \quad\left(\mathrm{NCH}_{2} \mathrm{CHCl}\right), \quad 63.3 \quad\left(\mathrm{CHClCH}_{2} \mathrm{CH}_{3}\right), \quad 81.0$ $\left(\mathrm{COOC}\left(\mathrm{CH}_{3}\right)_{3}\right), 127.3,128.4,128.9(\mathrm{CPh}), 139.1$ (ipso-CPh), $170.8(\mathrm{CO}) \mathrm{ppm}$. EIMS: $\mathrm{m} / z(\%)=$ 350 (13), 336 (35), 334 (100), 312 (11) $\left[\mathrm{MH}^{+}\right], 278$ (26), 256 (75), 254 (26), 242 (10), 220 (14). HRMS (ESI) calcd. for $\mathrm{C}_{17} \mathrm{H}_{27} \mathrm{ClNO}_{2}\left[\mathrm{MH}^{+}\right]: 312.1730$; found 312.1718.

$(R)-(+)-\{N$-[3-(4-benzyloxy-phenyl)-2-chloro-propyl]-benzylamino\}-acetic acid tertbutylester 28. (Table 1, Entry 8) Prepared according to GP-1. The pure compound was obtained after flash chromatography $\left(\mathrm{SiO}_{2}, \mathrm{PE} / \mathrm{AcOEt}, 9: 1\right)$ as colourless oil. Yield: $1.09 \mathrm{~g}(88 \%) ; R_{\mathrm{f}}=$ $0.80(\mathrm{PE} / \mathrm{AcOEt}, 9: 1) ;[\alpha]_{\mathrm{D}}{ }^{20}:+5.7\left(\mathrm{c}=0.20, \mathrm{CHCl}_{3}\right) .{ }^{1} \mathrm{H} \mathrm{NMR}\left(200 \mathrm{MHz}, \mathrm{CDCl}_{3}, 25{ }^{\circ} \mathrm{C}\right): 1.59$ $\left(\mathrm{s}, 9 \mathrm{H}, \mathrm{C}\left(\mathrm{CH}_{3}\right)_{3}\right), 2.89(\mathrm{dd}, J=14.3 \mathrm{~Hz}, J=8.6 \mathrm{~Hz}, 1 \mathrm{H}, \mathrm{A}$ of AB System, OPhCHHCHCl$), 3.18$ $\left(\mathrm{dd}, J=6.4 \mathrm{~Hz}, J=1.8 \mathrm{~Hz}, 2 \mathrm{H}, \mathrm{NCH}_{2} \mathrm{CHCl}\right), 3.35(\mathrm{dd}, J=14.3 \mathrm{~Hz}, J=4.4 \mathrm{~Hz}, 1 \mathrm{H}$, B of AB System, OPhCHHCHCl), 3.45 (s, 2H, $\left.\mathrm{NCH}_{2} \mathrm{COOtBu}\right), 4.01$ (s, 2H, NCH $\mathrm{Nh}_{2} \mathrm{Ph}, 4.05-4.27(\mathrm{~m}, 1 \mathrm{H}$, $\left.\mathrm{NCH}_{2} \mathrm{CHCl}\right), 5.12\left(\mathrm{~s}, 2 \mathrm{H}, \mathrm{OCH}_{2} \mathrm{Ph}\right), 7.02(\mathrm{~d}, J=8.6 \mathrm{~Hz}, 2 \mathrm{H}, \mathrm{OPh} H), 7.22(\mathrm{~d}, J=8.6 \mathrm{~Hz}, 2 \mathrm{H}$,

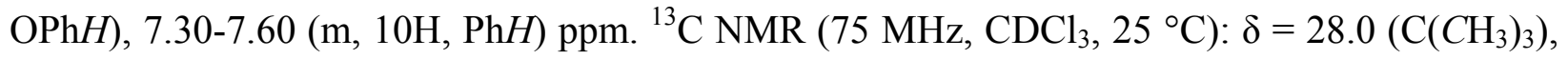
$41.1\left(\mathrm{PhCH}_{2} \mathrm{CHCl}\right), 55.7\left(\mathrm{NCH}_{2} \mathrm{CHCl}\right), 58.5\left(\mathrm{NCH}_{2} \mathrm{COO}^{t} \mathrm{Bu}\right), 60.6\left(\mathrm{NCH}_{2} \mathrm{CHCl}\right), 62.0$ $\left(\mathrm{NCH}_{2} \mathrm{Ph}\right), 69.7\left(\mathrm{OCH}_{2} \mathrm{Ph}\right), 80.8\left(\mathrm{COOC}_{\left.\left(\mathrm{CH}_{3}\right)_{3}\right), 114.5,127.1,127.3,127.7,128.2,128.4,128.7}\right.$ (CPh), 130.2, 137.0, 138.8, 157.4 (ipso-CPh), 170.4 (CO) ppm. EIMS: $m / z(\%)=480(100)$ $\left[\mathrm{MH}^{+}\right], 388(24), 378$ (10), 352 (11). HRMS (ESI) calcd. for $\mathrm{C}_{29} \mathrm{H}_{35} \mathrm{ClNO}_{3}\left[\mathrm{MH}^{+}\right]: 480.2305$; found 480.2308 .

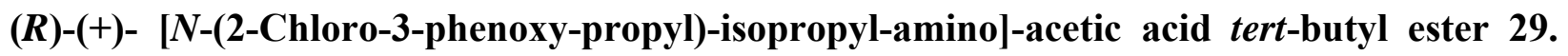
(Table 1, Entry 9) Prepared according to GP-1. The pure compound was obtained after flash chromatography $\left(\mathrm{SiO}_{2}, \mathrm{PE} / \mathrm{AcOEt}, 9: 1\right)$ as colourless oil. Yield: $300 \mathrm{mg}(85 \%) ; R_{\mathrm{f}}=0.50$ $(\mathrm{PE} / \mathrm{AcOEt}, 95: 5) ;[\alpha]_{\mathrm{D}}{ }^{20}:+19.5\left(\mathrm{c}=0.90, \mathrm{CHCl}_{3}\right) .{ }^{1} \mathrm{H} \mathrm{NMR}\left(200 \mathrm{MHz}, \mathrm{CDCl}_{3}, 25{ }^{\circ} \mathrm{C}\right): \delta=$ $1.02\left(\mathrm{~d}, J=4.6 \mathrm{~Hz}, 3 \mathrm{H}, \mathrm{NCHCH}_{3}\left(\mathrm{CH}_{3}\right)\right), 1.05$ (d, $J=4.8 \mathrm{~Hz}, 3 \mathrm{H}, \mathrm{NCHCH}_{3}\left(\mathrm{CH}_{3}\right), 1.45$ (s, 9H, $\left.\mathrm{C}\left(\mathrm{CH}_{3}\right)_{3}\right), 2.92-3.18\left(\mathrm{~m}, 3 \mathrm{H}, \mathrm{NCH}\left(\mathrm{CH}_{3}\right)_{2} \mathrm{NCH}_{2} \mathrm{CHCl}\right), 3.29(\mathrm{dd}, J=17.1 \mathrm{~Hz}, J=11.2 \mathrm{~Hz}, 2 \mathrm{H}$, $\mathrm{NCH}_{2} \mathrm{COOtBu}$ ), 4.15-4.28 (quintet, $\left.J=4.5 \mathrm{~Hz}, 1 \mathrm{H}, \mathrm{NCH}_{2} \mathrm{CH}(\mathrm{Cl}) \mathrm{CH}_{2}\right), 4.32(\mathrm{dd}, J=10.2 \mathrm{~Hz}, J$ $=3.9 \mathrm{~Hz}, 1 \mathrm{H}, \mathrm{A}$ of $\mathrm{AB}$ system, $\mathrm{PhOCHH}), 4.40(\mathrm{dd}, J=10.2 \mathrm{~Hz}, J=3.9 \mathrm{~Hz}, 1 \mathrm{H}, \mathrm{B}$ of AB system, $\mathrm{PhOCH} H)$, 6.90-7.02 (m, 3H, $\mathrm{Ph} H)$, 7.22-7.36 (m, 2H, $\mathrm{Ph} H)$ ppm. ${ }^{13} \mathrm{C}$ NMR $(75 \mathrm{MHz}$, $\left.\mathrm{CDCl}_{3}, 25{ }^{\circ} \mathrm{C}\right): \delta=19.1\left(\mathrm{NCHCH}_{3}\left(\mathrm{CH}_{3}\right)\right), 19.7\left(\mathrm{NCHCH}_{3}\left(\mathrm{CH}_{3}\right)\right), 28.1\left(\mathrm{C}_{\left.\left(\mathrm{CH}_{3}\right)_{3}\right), 53.2}\right.$ $\left(\mathrm{NCH}\left(\mathrm{CH}_{3}\right)_{2}\right), 54.0\left(\mathrm{NCH}_{2} \mathrm{CHCl}\right), 55.4\left(\mathrm{NCH}_{2} \mathrm{COOtBu}\right), 58.5\left(\mathrm{NCH}_{2} \mathrm{CHCl}\right), 69.3\left(\mathrm{OCH}_{2} \mathrm{CHCl}\right)$, $80.7\left(\mathrm{COOC}\left(\mathrm{CH}_{3}\right)_{3}\right), 114.8,121.1,129.5(\mathrm{CPh}), 158.5$ (ipso-CPh), $171.7(\mathrm{CO}) \mathrm{ppm}$. EIMS: $\mathrm{m} / z$ $(\%)=364(90)(\mathrm{M}+\mathrm{Na})^{+}, 346(100), 308$ (45), 290 (82), 286 (72), 272 (23). HRMS (ESI) calcd. for $\mathrm{C}_{18} \mathrm{H}_{29} \mathrm{ClNO}_{3}\left[\mathrm{MH}^{+}\right]$: 342.1836; found 342.1828. 
(R)-(+)-[N-(2-Chloro-3-phenoxy-propyl)-benzyl-amino]-acetic acid tert-butyl ester 30. (Table 1, Entry 10) Prepared according to GP-1. The pure compound was obtained after flash chromatography $\left(\mathrm{SiO}_{2}, \mathrm{PE} / \mathrm{AcOEt}, 9: 1\right)$ as colourless oil. Yield: $300 \mathrm{mg}(64 \%) ; R_{\mathrm{f}}=0.45$ $(\mathrm{PE} / \mathrm{AcOEt}, 95: 5) ;[\alpha]_{\mathrm{D}}{ }^{20}:+10.6\left(\mathrm{c}=0.80, \mathrm{CHCl}_{3}\right) .{ }^{1} \mathrm{H} \mathrm{NMR}\left(300 \mathrm{MHz}, \mathrm{CDCl}_{3}, 25{ }^{\circ} \mathrm{C}\right): 1.48(\mathrm{~s}$, 9H, $\left.\mathrm{C}\left(\mathrm{CH}_{3}\right)_{3}\right), 3.06(\mathrm{dd}, J=14.2 \mathrm{~Hz}, J=5.3 \mathrm{~Hz}, 1 \mathrm{H}, \mathrm{NCHHCHCl}), 3.19(\mathrm{dd}, J=14.2 \mathrm{~Hz}, J=$ $5.3 \mathrm{~Hz}, 1 \mathrm{H}, \mathrm{NCH} H \mathrm{CHCl}), 3.32$ (s, 2H, $\left.\mathrm{NCH}_{2} \mathrm{COOtBu}\right), 3.92$ (s, 2H, $\mathrm{NCH}_{2} \mathrm{Ph}$ ), 4.03-4.13 (m, $\left.2 \mathrm{H}, \mathrm{NCH}_{2} \mathrm{CHCl}, \mathrm{PhOCHH}\right), 4.14-4.22(\mathrm{~m}, 1 \mathrm{H}, \mathrm{PhOCH} H), 6.82-7.04(\mathrm{~m}, 3 \mathrm{H}, \mathrm{OPhH}), 7.10-7.30$

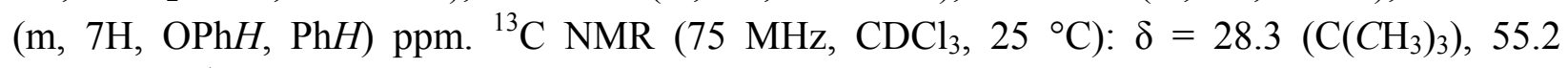
$\left(\mathrm{NCH}_{2} \mathrm{COO}^{\mathrm{t}} \mathrm{Bu}\right), 56.8\left(\mathrm{NCH}_{2} \mathrm{CHCl}\right), 58.1\left(\mathrm{NCH}_{2} \mathrm{CHCl}\right), 59.0\left(\mathrm{NCH}_{2} \mathrm{Ph}\right), 70.1\left(\mathrm{PhOCH}_{2} \mathrm{CHCl}\right)$, $81.1\left(\mathrm{COOC}\left(\mathrm{CH}_{3}\right)_{3}\right), 114.8,121.0,126.2,128.5,129.0,129.5(C \mathrm{Ph}), 158.0$ (ipso-CPh), 171.0 (CO) ppm. EIMS: $m / z(\%)=390(100)\left[\mathrm{MH}^{+}\right], 362(30), 354$ (56), 334 (99), 288 (24), 234 (10). HRMS (ESI) calcd. for $\mathrm{C}_{22} \mathrm{H}_{29} \mathrm{ClNO}_{3}\left[\mathrm{MH}^{+}\right]$: 390.1836; found 390.1830.

2R,3R)-(-)-[(3-Benzyloxy-2-chloro-butyl)-benzyl amino]-acetic acid tert-butyl ester 31. (Table 1, Entry 11) Prepared according to GP-1. The pure compound was obtained after flash chromatography $\left(\mathrm{SiO}_{2}, \mathrm{PE} / \mathrm{AcOEt}, 9: 1\right)$ as colourless oil. Yield: $179 \mathrm{mg}(95 \%) ; R_{\mathrm{f}}=0.82$ $(\mathrm{PE} / \mathrm{AcOEt}, 9: 1) ;[\alpha]_{\mathrm{D}}{ }^{20}:-11.9\left(\mathrm{c}=0.80, \mathrm{CHCl}_{3}\right) .{ }^{1} \mathrm{H} \mathrm{NMR}\left(200 \mathrm{MHz}, \mathrm{CDCl}_{3}, 25^{\circ} \mathrm{C}\right): 1.22(\mathrm{~d}, J$ $\left.=6.2 \mathrm{~Hz}, 3 \mathrm{H}, \mathrm{CH}_{3}\right), 1.51\left(\mathrm{~s}, 9 \mathrm{H}, \mathrm{C}\left(\mathrm{CH}_{3}\right)_{3}\right), 3.07(\mathrm{dd}, J=14.3 \mathrm{~Hz}, J=7.0 \mathrm{~Hz}, 1 \mathrm{H}, \mathrm{NCHHCHCl})$, $3.15(\mathrm{dd}, J=14.3 \mathrm{~Hz}, J=6.6 \mathrm{~Hz}, 2 \mathrm{H}, \mathrm{NCH} H \mathrm{CHCl})$ ), 3.30 (s, 2H, NCH $\mathrm{COO}^{t} \mathrm{Bu}$ ), 3.79-3.98 (m, $\left.3 \mathrm{H}, \mathrm{NCH}_{2} \mathrm{Ph}, \mathrm{CH}_{3} \mathrm{CHOCH}_{2} \mathrm{Ph}\right), 4.14-4.25\left(\mathrm{~m}, 1 \mathrm{H}, \mathrm{NCH}_{2} \mathrm{CHCl}\right), 4.54(\mathrm{~d}, J=12.1 \mathrm{~Hz}, 1 \mathrm{H}, \mathrm{A}$ of AB system, PhOCHHPh), $4.61(\mathrm{~d}, J=12.1 \mathrm{~Hz}, 1 \mathrm{H}, \mathrm{B}$ of AB system, PhOCHHPh), 7.23-7.42 $(\mathrm{m}, 10 \mathrm{H}, \mathrm{Ph} H)$ ppm. ${ }^{13} \mathrm{C} \mathrm{NMR}\left(75 \mathrm{MHz}, \mathrm{CDCl}_{3}, 25^{\circ} \mathrm{C}\right): \delta=14.9\left(\mathrm{CH}_{3}\right), 28.1\left(\mathrm{C}_{\left.\left(\mathrm{CH}_{3}\right)_{3}\right), 55.5}\right.$ $\left(\mathrm{NCH}_{2} \mathrm{CHCl}\right), 57.5\left(\mathrm{NCH}_{2} \mathrm{COO}^{t} \mathrm{Bu}\right), 58.5\left(\mathrm{NCH}_{2} \mathrm{CHCl}\right), 63.4\left(\mathrm{NCH}_{2} \mathrm{Ph}\right), 70.6\left(\mathrm{PhCH}_{2} \mathrm{O}\right), 74.9$ $\left(\mathrm{CH}_{3} \mathrm{CHOCH}_{2} \mathrm{Ph}\right), 80.9\left(\mathrm{COOC}\left(\mathrm{CH}_{3}\right)_{3}\right), 127.2,127.5,127.6,128.3,128.8(\mathrm{CPh}), 138.2,138.7$ (ipso-CPh), $170.4(\mathrm{CO})$ ppm. EIMS: $m / z(\%)=440(100)(\mathrm{M}+\mathrm{Na})^{+}, 384(32), 362(19)$. HRMS (ESI) calcd. for $\mathrm{C}_{24} \mathrm{H}_{33} \mathrm{ClNO}_{3}\left[\mathrm{MH}^{+}\right]$: 418.2149; found 418.2138.

\section{General procedure for the synthesis of azetidines (GP-2)}

To a solution of chloride $(1 \mathrm{mmol})$ in pure THF $(10 \mathrm{~mL}$ for chloro nitriles) or $8 / 2 \mathrm{THF} / \mathrm{HMPA}$ (for chloro esters) was added dropwise at $-90^{\circ} \mathrm{C}$ a solution of LiHMDS (1M solution in THF, $1.5 \mathrm{~mL}, 1.5 \mathrm{mmol})$. The reaction was monitored by TLC and then quenched after completion by the addition of an aqueous saturated solution of $\mathrm{NH}_{4} \mathrm{Cl}$ at $0{ }^{\circ} \mathrm{C}$. Extraction of the reaction mixture using ether followed by usual workup gave azetidine 34-45.

(2R and 2S, 3S)1-Benzyl-3-isobutyl-azetidine-2-carbonitrile 34 and 35. (Table 2, Entry 1) Prepared according to GP-2 and flash chromatographic purification of the reaction mixture gave an inseparable mixture of cis and trans diasteromers (3:2) $\left(\mathrm{SiO}_{2}\right.$, PE/AcOEt, 95:5) as colourless oil. Yield: $103 \mathrm{mg}(95 \%) ; R_{\mathrm{f}}=0.60$ (PE/AcOEt, 9:1); ${ }^{1} \mathrm{H} \mathrm{NMR}\left(200 \mathrm{MHz}, \mathrm{CDCl}_{3}, 25{ }^{\circ} \mathrm{C}\right.$, mixture of cis and trans diasteromers (3:2)): $0.94\left(\mathrm{~d}, J=6.2 \mathrm{~Hz}, 6 \mathrm{H},\left(\mathrm{CH}_{3}\right)_{2} \mathrm{CH}\right), 1.46-1.70(\mathrm{~m}$, $3 \mathrm{H},\left(\mathrm{CH}_{3}\right)_{2} \mathrm{CHCH}_{2}$ ), 2.72 (Appt triplet, $J=7.3 \mathrm{~Hz} 1 \mathrm{H}, \mathrm{H}-4$, cis-isomer), 2.77-2.94 (m, 1H, H-3), 3.02 (Appt triplet, $J=6.8 \mathrm{~Hz} 1 \mathrm{H}, \mathrm{H}-4^{\prime}$, cis-isomer), 3.38 (Appt triplet, $J=6.7 \mathrm{~Hz}, 1 \mathrm{H}, \mathrm{H}-4$, 
trans-isomer), 3.47 (d, $J=7.0 \mathrm{~Hz}, 1 \mathrm{H}, \mathrm{H}-2$, cis-isomer), 3.53 (Appt triplet, $J=7.0 \mathrm{~Hz}, 1 \mathrm{H}, \mathrm{H}-4^{\prime}$, trans-isomer), 3.54 (d, $J=12.8 \mathrm{~Hz}, 1 \mathrm{H}$, A of AB system, NCHHPh ), 3.71 (d, $J=12.8 \mathrm{~Hz}, 1 \mathrm{H}$, $\mathrm{B}$ of AB system, $\mathrm{NCH} H \mathrm{Ph}), 4.19$ (d, $J=7.3 \mathrm{~Hz}, 1 \mathrm{H}, \mathrm{H}-2$, trans-isomer), 7.14-7.46 (m, $5 \mathrm{H}, \mathrm{Ph} H$ ) ppm. ${ }^{13} \mathrm{C}$ NMR $\left(50 \mathrm{MHz}, \mathrm{CDCl}_{3}, 25{ }^{\circ} \mathrm{C}\right.$, cis-isomer $): \delta=22.4\left(2 \times \mathrm{CH}_{3}\right), 26.5\left(\left(\mathrm{CH}_{3}\right)_{2} \mathrm{CH}\right), 32.2$ $(\mathrm{C}-3), 42.5\left(\left(\mathrm{CH}_{3}\right)_{2} \mathrm{CHCH}_{2}\right), 57.3(\mathrm{C}-2), 58.8(\mathrm{C}-4), 61.8\left(\mathrm{NCH}_{2} \mathrm{Ph}\right), 116.8(\mathrm{CN}), 127.5,128.5$, $128.8(C \mathrm{Ph}), 136.6\left(\right.$ ipso-CPh) ppm. CIMS: $m / z(\%)=229(89)\left[\mathrm{MH}^{+}\right], 202(18), 172$ (5), 137 (45), 91 (100). HRMS (ESI) calcd. for $\mathrm{C}_{15} \mathrm{H}_{21} \mathrm{~N}_{2}\left[\mathrm{MH}^{+}\right]$: 229.1699; found: 229.1697.

(2S,3S)-(-)-1-Benzyl-3-methylazetidine-2-carbonitrile 36 and (2R,3S)-(+)-1-benzyl-3methylazetidine-2-carbonitrile 37. (Table 2, Entry 2) Prepared according to GP-2. Analytical samples of each diastereoisomer could be obtained after flash chromatography $\left(\mathrm{SiO}_{2}, \mathrm{PE} / \mathrm{AcOEt}\right.$, 9:1) as colourless oils. Overall yield: $179 \mathrm{mg}$ (60\%). Compound 36 (2,3-trans isomer) $R_{\mathrm{f}}=0.75$ (PE/AcOEt, 9:1); $[\alpha]_{578}{ }^{20}:-48.2$ (c = 0.40, $\left.\mathrm{CHCl}_{3}\right) .{ }^{1} \mathrm{H} \mathrm{NMR}\left(200 \mathrm{MHz}, \mathrm{CDCl}_{3}, 25{ }^{\circ} \mathrm{C}\right): 1.25$ (d, $\left.J=6.6 \mathrm{~Hz}, 3 \mathrm{H}, \mathrm{CH}_{3}\right), 2.75(\mathrm{dd}, J=13.6 \mathrm{~Hz}, J=7.2 \mathrm{~Hz}, 1 \mathrm{H}, \mathrm{H}-4), 2.86$ (septet, $J=6.8 \mathrm{~Hz}, 1 \mathrm{H}$, H-3), 3.45 (d, $J=6.8 \mathrm{~Hz}, 1 \mathrm{H}, \mathrm{H}-2$ ), 3.53 (Appt triplet, $J=6.6 \mathrm{~Hz}, 1 \mathrm{H}, \mathrm{H}-4$ ) $) 3.64$ (d, $J=12.7$ $\mathrm{Hz}, 1 \mathrm{H}, \mathrm{A}$ of AB system, NCHHPh), $3.76(\mathrm{~d}, J=12.7 \mathrm{~Hz}, 1 \mathrm{H}, \mathrm{B}$ of AB system, NCHHPh ), 7.22-7.42 (m, 5H, $\mathrm{Ph} H$ ) ppm. ${ }^{13} \mathrm{C}$ NMR (50 MHz, $\left.\mathrm{CDCl}_{3}, 25{ }^{\circ} \mathrm{C}\right): \delta=18.0\left(\mathrm{CH}_{3}\right), 32.5(\mathrm{C}-3)$, $58.1(\mathrm{C}-4), 59.5(\mathrm{C}-2), 61.5\left(\mathrm{NCH}_{2} \mathrm{Ph}\right), 118.8(\mathrm{CN}), 127.6,128.4,128.5,128.8(\mathrm{CPh}), 136.2$ (ipso-CPh) ppm. EIMS: $m / z(\%)=187$ (19) $\left[\mathrm{MH}^{+}\right], 161$ (13), 160 (100), 159 (23), 91 (3). HRMS (ESI) calcd. for $\mathrm{C}_{12} \mathrm{H}_{15} \mathrm{~N}_{2}\left[\mathrm{MH}^{+}\right]$: 187.1235; found 187.1240. Compound 37 (2,3-cis isomer): $R_{\mathrm{f}}$ $=0.72(\mathrm{PE} / \mathrm{AcOEt}, 9: 1) ;[\alpha]_{578}{ }^{20}:+76.1\left(\mathrm{c}=0.90, \mathrm{CHCl}_{3}\right) .{ }^{1} \mathrm{H} \mathrm{NMR}\left(200 \mathrm{MHz}, \mathrm{CDCl}_{3}, 25{ }^{\circ} \mathrm{C}\right)$ : $1.38\left(\mathrm{~d}, J=6.8 \mathrm{~Hz}, 3 \mathrm{H}, \mathrm{CH}_{3}\right.$ ), 2.82 (septet, $J=7.2 \mathrm{~Hz}, 1 \mathrm{H}, \mathrm{H}-3$ ), 2.99 (Appt triplet, $J=6.5 \mathrm{~Hz}$, 1H, H-4), 3.32 (Appt triplet, $J=6.5 \mathrm{~Hz}, 1 \mathrm{H}, \mathrm{H}-4$ ), 3.69 (d, $J=12.7 \mathrm{~Hz}, 1 \mathrm{H}$, A of AB system, $\mathrm{NCHHPh}), 3.76(\mathrm{~d}, J=12.7 \mathrm{~Hz}, 1 \mathrm{H}, \mathrm{B}$ of AB system, $\mathrm{NCH} H \mathrm{Ph}), 4.11(\mathrm{~d}, J=7.5 \mathrm{~Hz}, 1 \mathrm{H}, \mathrm{H}-2)$, 7.21-7.41 (m, 5H, $\mathrm{Ph} H)$ ppm. ${ }^{13} \mathrm{C}$ NMR $\left(50 \mathrm{MHz}, \mathrm{CDCl}_{3}, 25{ }^{\circ} \mathrm{C}\right): \delta=16.2\left(\mathrm{CH}_{3}\right), 28.4(\mathrm{C}-3)$, $57.2(\mathrm{C}-4), 59.2(\mathrm{C}-2), 59.9\left(\mathrm{NCH}_{2} \mathrm{Ph}\right), 127.5,128.5,128.8(C \mathrm{Ph}, C \mathrm{~N}), 136.4$ (ipso-CPh) ppm. EIMS: $m / z(\%)=187(8)\left[\mathrm{MH}^{+}\right], 161(15), 160(100), 159$ (21), 91 (6). HRMS (ESI) calcd. for $\mathrm{C}_{12} \mathrm{H}_{15} \mathrm{~N}_{2}\left[\mathrm{MH}^{+}\right]: 187.1235$; found 187.1238.

(2R and 2S, 3S)-1-Benzyl-3-isopropyl-azetidine-2-carbonitrile 38 and 39. (Table 2, Entry 3) Prepared according to GP-2 and flash chromatographic purification of the reaction mixture gave an inseparable mixture of $c$ is and trans diasteromers (3:2) $\left(\mathrm{SiO}_{2}, \mathrm{PE} / \mathrm{AcOEt}, 95: 5\right)$ as colourless oil. Yield: $20 \mathrm{mg}(25 \%) ; R_{\mathrm{f}}=0.44$ (PE/AcOEt, 9:1); ${ }^{1} \mathrm{H} \mathrm{NMR}\left(300 \mathrm{MHz}, \mathrm{CDCl}_{3}, 25{ }^{\circ} \mathrm{C}\right.$, mixture of cis and trans diasteromers (3:2)): $0.70\left(\mathrm{~d}, J=6.6 \mathrm{~Hz}, 3 \mathrm{H}, \mathrm{CH}_{3}\right), 0.80\left(\mathrm{~d}, J=6.5 \mathrm{~Hz}, 3 \mathrm{H}, \mathrm{CH}_{3}\right)$, 1.50-1.65 (m, 1H, $\left(\mathrm{CH}_{3}\right)_{2} \mathrm{CH}$, cis-isomer), 1.80-1.96 (m, $1 \mathrm{H},\left(\mathrm{CH}_{3}\right)_{2} \mathrm{CH}$, trans-isomer), 2.22-2.40 (m, 1H, H-3), 2.65 (Appt triplet, $J=7.3 \mathrm{~Hz}, 1 \mathrm{H}, \mathrm{H}-4$, cis-isomer), 2.92 (Appt triplet, $J=7.5 \mathrm{~Hz}$, 1H, H-4 , cis-isomer), 3.32 (Appt triplet, $J=7.1 \mathrm{~Hz}, 1 \mathrm{H}, \mathrm{H}-4$, trans-isomer), 3.39 (Appt triplet, $J$ $=7.1 \mathrm{~Hz}, 1 \mathrm{H}, \mathrm{H}-4^{\prime}$, trans-isomer), 3.41 (d, $J=7.3 \mathrm{~Hz}, 1 \mathrm{H}, \mathrm{H}-2$, cis-isomer), 3.48 (d, $J=12.5$ $\mathrm{Hz}, 1 \mathrm{H}, \mathrm{A}$ of AB system, NCHHPh ), $3.62(\mathrm{~d}, J=12.5 \mathrm{~Hz}, 1 \mathrm{H}, \mathrm{B}$ of AB system, NCHHPh ), 4.10 (d, $J=7.3 \mathrm{~Hz}, 1 \mathrm{H}, \mathrm{H}-2$, trans-isomer), 7.04-7.30 (m, 5H, $\mathrm{PhH}) \mathrm{ppm} .{ }^{13} \mathrm{C} \mathrm{NMR} \mathrm{(75} \mathrm{MHz,}$ $\mathrm{CDCl}_{3}, 25^{\circ} \mathrm{C}$, cis-isomer): $\delta=20.3\left(\mathrm{CH}_{3}\right), 20.9\left(\mathrm{CH}_{3}\right), 32.7\left(\left(\mathrm{CH}_{3}\right)_{2} \mathrm{CH}\right), 44.5(\mathrm{C}-3), 56.8(\mathrm{C}-2)$, $57.9(\mathrm{C}-4), 61.8\left(\mathrm{NCH}_{2} \mathrm{Ph}\right), 119.3(\mathrm{CN}), 127.5,128.1,128.5(C \mathrm{Ph}), 137.4$ (ipso-CPh) ppm. 
CIMS: $m / z(\%)=214(8)\left[\mathrm{M}^{+}\right], 213$ (11), 190 (13), 188 (100), 121 (14). HRMS (ESI) calcd. for $\mathrm{C}_{14} \mathrm{H}_{19} \mathrm{~N}_{2}\left[\mathrm{MH}^{+}\right]: 215.1548$; found 215.1536 .

(2R, 3S)-(+)-1-Benzyl-3-isobutyl-azetidine-2-carboxylic acid tert-butyl ester 40. (Table 2, Entry 5) Prepared according to GP-2. The pure compound was obtained after flash chromatography $\left(\mathrm{SiO}_{2}, \mathrm{PE} / \mathrm{AcOEt}, 9: 1\right)$ as colourless oil. Yield: $131 \mathrm{mg}(73 \%) ; R_{\mathrm{f}}=0.82$ (PE/AcOEt, 9:1); $[\alpha]_{365}{ }^{20}:+197.0\left(\mathrm{c}=0.70, \mathrm{CHCl}_{3}\right) .{ }^{1} \mathrm{H} \mathrm{NMR}\left(300 \mathrm{MHz}, \mathrm{CDCl}_{3}, 25{ }^{\circ} \mathrm{C}\right): 0.73$ $\left(\mathrm{d}, J=6.7 \mathrm{~Hz}, 3 \mathrm{H}, \mathrm{CH}_{3}\right), 0.79\left(\mathrm{~d}, J=6.7 \mathrm{~Hz}, 3 \mathrm{H}, \mathrm{CH}_{3}\right), 1.31\left(\mathrm{~s}, 9 \mathrm{H}, \mathrm{C}\left(\mathrm{CH}_{3}\right)_{3}\right), 1.32-1.63(\mathrm{~m}, 3 \mathrm{H}$, $\left.\mathrm{CH}\left(\mathrm{CH}_{3}\right)_{2}, \mathrm{CH}_{2} \mathrm{CH}\left(\mathrm{CH}_{3}\right)_{2}\right), 2.49-2.68(\mathrm{~m}, 1 \mathrm{H}, \mathrm{H}-3), 2.95$ (Appt triplet, $J=7.0 \mathrm{~Hz}, 1 \mathrm{H}, \mathrm{H}-4$ ), $3.15\left(\mathrm{dd}, J=6.6 \mathrm{~Hz}, J=3.1 \mathrm{~Hz}, 1 \mathrm{H}, \mathrm{H}-4^{\prime}\right), 3.54(\mathrm{~d}, J=12.7 \mathrm{~Hz}, 1 \mathrm{H}, \mathrm{A}$ of AB system, $\mathrm{NCHHPh}), 3.63(\mathrm{~d}, J=12.7 \mathrm{~Hz}, 1 \mathrm{H}, \mathrm{B}$ of AB system, NCHHPh ), 3.66 (d, J=8.5 Hz, $1 \mathrm{H}, \mathrm{H}-2)$, 7.14-7.32 (m, 5H, $\mathrm{PhH}) .{ }^{13} \mathrm{C} \mathrm{NMR}\left(75 \mathrm{MHz}, \mathrm{CDCl}_{3}, 25{ }^{\circ} \mathrm{C}\right): \delta=21.9\left(\mathrm{CH}_{3}\right), 23.2\left(\mathrm{CH}_{3}\right), 26.0$ $\left(\mathrm{CH}\left(\mathrm{CH}_{3}\right)_{2}\right), 28.1\left(\mathrm{COOC}\left(\mathrm{CH}_{3}\right)_{3}\right), 31.9(\mathrm{C}-3), 38.3\left(\mathrm{CH}_{2} \mathrm{CH}\left(\mathrm{CH}_{3}\right)_{2}\right), 55.4(\mathrm{C}-4), 61.5\left(\mathrm{NCH}_{2} \mathrm{Ph}\right)$, $67.3(\mathrm{C}-2), 80.7\left(\mathrm{COOC}\left(\mathrm{CH}_{3}\right)_{3}\right), 127.1,128.2,129.3(\mathrm{CPh}), 137.4$ (ipso-CPh), $170.8(\mathrm{CO}) \mathrm{ppm}$. CIMS: $m / z(\%)=304(24)\left[\mathrm{MH}^{+}\right], 248$ (13), 202 (100), 91 (54). HRMS (ESI) calcd. for $\mathrm{C}_{19} \mathrm{H}_{30} \mathrm{NO}_{2}\left[\mathrm{MH}^{+}\right]: 304.2277$; found 304.2271.

(2R, 3S)-(+)-1-Benzyl-3-methyl-azetidine-2-carboxylic acid tert-butyl ester 41. (Table 2, Entry 6) Prepared according to GP-2. The pure compound was obtained by flash chromatography $\left(\mathrm{SiO}_{2}, \mathrm{PE} / \mathrm{AcOEt}, 9: 1\right)$ as colourless oil. Yield: $1.050 \mathrm{~g}(80 \%) ; R_{\mathrm{f}}=0.80(\mathrm{PE} / \mathrm{AcOEt}, 9: 1) ;[\alpha]_{\mathrm{D}}{ }^{20}$ : $+91.3\left(\mathrm{c}=0.40, \mathrm{CHCl}_{3}\right) .{ }^{1} \mathrm{H} \mathrm{NMR}\left(200 \mathrm{MHz}, \mathrm{CDCl}_{3}, 25^{\circ} \mathrm{C}\right): 1.23\left(\mathrm{~d}, J=7.0 \mathrm{~Hz}, 3 \mathrm{H}, \mathrm{CH}_{3}\right)$, 1.40 (s, $\left.9 \mathrm{H}, \mathrm{C}\left(\mathrm{CH}_{3}\right)_{3}\right), 2.58-2.69$ (m, 1H, H-3), 2.90-3.10 (m, 2H, H-4, H-4'), 3.54 (d, J= 12.5 $\mathrm{Hz}, 1 \mathrm{H}, \mathrm{A}$ of AB system, NCHHPh), $3.68(\mathrm{~d}, J=8.0 \mathrm{~Hz}, 1 \mathrm{H}, \mathrm{H}-2), 3.74(\mathrm{~d}, J=12.5 \mathrm{~Hz}, 1 \mathrm{H}, \mathrm{B}$ of $\mathrm{AB}$ system, $\mathrm{NCH} H \mathrm{Ph}), 7.18-7.39(\mathrm{~m}, 5 \mathrm{H}, \mathrm{Ph} H) .{ }^{13} \mathrm{C} \mathrm{NMR}\left(75 \mathrm{MHz}, \mathrm{CDCl}_{3}, 25{ }^{\circ} \mathrm{C}\right): \delta=15.5$ $\left(\mathrm{CH}_{3}\right), 28.2\left(\mathrm{COOC}\left(\mathrm{CH}_{3}\right)_{3}\right), 28.6(\mathrm{C}-3), 56.8(\mathrm{C}-4), 61.6\left(\mathrm{NCH}_{2} \mathrm{Ph}\right), 67.3(\mathrm{C}-2), 80.6$ $\left(\mathrm{COOC}\left(\mathrm{CH}_{3}\right)_{3}\right), 127.0,128.2,129.2(\mathrm{CPh}), 137.5($ ipso-CPh), $170.8(\mathrm{CO}) \mathrm{ppm}$. CIMS: $\mathrm{m} / z(\%)=$ 300 (10), 284 (74), 262 (28) $\left[\mathrm{MH}^{+}\right], 206$ (100). HRMS (ESI) calcd. for $\mathrm{C}_{16} \mathrm{H}_{24} \mathrm{NO}_{2}\left[\mathrm{MH}^{+}\right]$: 262.1802; found 262.1796.

(2S, 3R)-(-)-1-Benzyl-3-ethyl-azetidine-2-carboxylic acid tert-butyl ester 42. (Table 2, Entry 7) Prepared according to GP-2. The pure compound was obtained after flash chromatography $\left(\mathrm{SiO}_{2}, \mathrm{PE} / \mathrm{AcOEt}, 9: 1\right)$ as colourless oil. Yield: $272 \mathrm{mg}(85 \%) ; R_{\mathrm{f}}=0.75(\mathrm{PE} / \mathrm{AcOEt}, 9: 1)$; $[\alpha]_{\mathrm{D}}{ }^{20}:-91.1\left(\mathrm{c}=0.90, \mathrm{CHCl}_{3}\right) .{ }^{1} \mathrm{H} \mathrm{NMR}\left(300 \mathrm{MHz}, \mathrm{CDCl}_{3}, 25{ }^{\circ} \mathrm{C}\right): 0.75(\mathrm{t}, J=7.3 \mathrm{~Hz}, 3 \mathrm{H}$, $\left.\mathrm{CH}_{3}\right), 1.30$ (s, 9H, C(CH3) $)_{3}$, 1.49-1.68 (m, 2H, $\left.\mathrm{CH}_{2} \mathrm{CH}_{3}\right), 2.30-2.46$ (m, $\left.1 \mathrm{H}, \mathrm{H}-3\right), 2.89$ (Appt triplet, $J=7.1 \mathrm{~Hz}, 1 \mathrm{H}, \mathrm{H}-4), 3.01$ (dd, $\left.J=6.6 \mathrm{~Hz}, J=2.8 \mathrm{~Hz}, 1 \mathrm{H}, \mathrm{H}-4^{\prime}\right), 3.52$ (d, $J=12.5 \mathrm{~Hz}$, 1H, A of AB system, NCHHPh), 3.63 (d, $J=12.5 \mathrm{~Hz}, 1 \mathrm{H}, \mathrm{B}$ of AB system, $\mathrm{NCH} H \mathrm{Ph}$ ), 3.64 (d, $J=7.0 \mathrm{~Hz}, 1 \mathrm{H}, \mathrm{H}-2), 7.24-7.51(\mathrm{~m}, 5 \mathrm{H}, \mathrm{Ph} H) .{ }^{13} \mathrm{C} \mathrm{NMR}\left(75 \mathrm{MHz}, \mathrm{CDCl}_{3}, 25{ }^{\circ} \mathrm{C}\right): \delta=11.4$ $\left(\mathrm{CH}_{3}\right), 22.4\left(\mathrm{CH}_{2} \mathrm{CH}_{3}\right), 28.1\left(\mathrm{COOC}\left(\mathrm{CH}_{3}\right)_{3}\right), 35.5(\mathrm{C}-3), 54.7(\mathrm{C}-4), 61.7\left(\mathrm{NCH}_{2} \mathrm{Ph}\right), 67.2(\mathrm{C}-2)$,

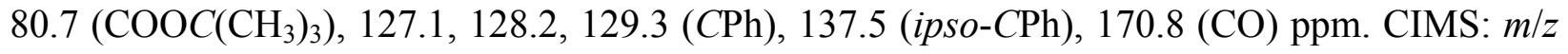
$(\%)=298$ (100), 242 (37), 220 (34). HRMS (ESI) calcd. for $\mathrm{C}_{17} \mathrm{H}_{26} \mathrm{NO}_{2}\left[\mathrm{MH}^{+}\right]$: 276.1964; found 276.1954 .

(2R, 3S)-(+)-1-Benzyl-3-(4-benzyloxy-benzyl)-azetidine-2-carboxylic acid tert-butyl ester 43. (Table 2, Entry 8) Prepared according to GP-2. The pure compound was obtained after flash chromatography $\left(\mathrm{SiO}_{2}, \mathrm{PE} / \mathrm{AcOEt}, 9: 1\right)$ as colourless oil. Yield: $93 \mathrm{mg}(50 \%) ; R_{\mathrm{f}}=0.70$ 
(PE/AcOEt, 9:1); $[\alpha]_{\mathrm{D}}{ }^{20}:+160.0\left(\mathrm{c}=0.30, \mathrm{CHCl}_{3}\right) .{ }^{1} \mathrm{H} \mathrm{NMR}\left(300 \mathrm{MHz}, \mathrm{CDCl}_{3}, 25{ }^{\circ} \mathrm{C}\right): 1.32(\mathrm{~s}$, 9H, $\left.\mathrm{C}\left(\mathrm{CH}_{3}\right)_{3}\right), 2.62-2.90(\mathrm{~m}, 5 \mathrm{H}, \mathrm{CHCH} 2 \mathrm{Ph}, \mathrm{H}-3, \mathrm{H}-4), 3.51(\mathrm{~d}, J=12.5 \mathrm{~Hz}, 1 \mathrm{H}, \mathrm{A}$ of AB system, $\mathrm{NCHHPh}), 3.66(\mathrm{~d}, J=13.0 \mathrm{~Hz}, 1 \mathrm{H}, \mathrm{B}$ of AB system, $\mathrm{NCH} H \mathrm{Ph}$ ), 3.69 (d, $J=8.3 \mathrm{~Hz}$, $1 \mathrm{H}, \mathrm{H}-2), 4.92\left(\mathrm{~s}, 2 \mathrm{H}, \mathrm{PhCH}_{2} \mathrm{O}\right), 6.77(\mathrm{~d}, J=8.5 \mathrm{~Hz}, 2 \mathrm{H}, \mathrm{OPh} H), 6.98(\mathrm{~d}, J=8.5 \mathrm{~Hz}, 2 \mathrm{H}$, $\mathrm{OPh} H), 7.18-7.39(\mathrm{~m}, 10 \mathrm{H}, \mathrm{Ph} H) .{ }^{13} \mathrm{C} \mathrm{NMR}\left(75 \mathrm{MHz}, \mathrm{CDCl}_{3}, 25{ }^{\circ} \mathrm{C}\right): \delta=28.2\left(\mathrm{COOC}\left(\mathrm{CH}_{3}\right)_{3}\right)$, $34.4\left(\mathrm{CHCH}_{2} \mathrm{PhO}\right), 34.8(\mathrm{C}-3), 54.2(\mathrm{C}-4), 61.7\left(\mathrm{NCH}_{2} \mathrm{Ph}\right), 66.8(\mathrm{C}-2), 70.0\left(\mathrm{OCH}_{2} \mathrm{Ph}\right), 81.1$ $\left(\mathrm{COOC}\left(\mathrm{CH}_{3}\right)_{3}\right)$, 114.8, 127.2, 127.5, 127.7, 128.0, 128.3, 128.6, $129.4(\mathrm{CPh}), 129.8,132.2$, 137.2, 157.3 (ipso-CPh), 170.7 (CO) ppm. CIMS: $m / z(\%)=444$ (100) $\left[\mathrm{MH}^{+}\right], 419$ (7), 388 (7), 354 (7), 342 (30). HRMS (ESI) calcd. for $\mathrm{C}_{29} \mathrm{H}_{34} \mathrm{NO}_{3}\left[\mathrm{MH}^{+}\right]$: 444.2539; found 444.2542.

(2R, 3S)-(+)-1-Isopropyl-3-phenoxymethyl-azetidine-2-carboxylic acid tert-butyl ester 44. (Table 2, Entry 9) Prepared according to GP-2. The pure compound was obtained after flash chromatography $\left(\mathrm{SiO}_{2}, \mathrm{PE} / \mathrm{AcOEt}, 4: 1\right)$ as colourless oil. Yield: $40 \mathrm{mg}(50 \%) ; R_{\mathrm{f}}=0.80$ (PE/AcOEt, 4:1); $[\alpha]_{\mathrm{D}}{ }^{20}:+50.8\left(\mathrm{c}=1.30, \mathrm{CHCl}_{3}\right) .{ }^{1} \mathrm{H}$ NMR $\left(200 \mathrm{MHz}, \mathrm{CDCl}_{3}, 25^{\circ} \mathrm{C}\right): 0.93(\mathrm{~d}$, $\left.J=6.4 \mathrm{~Hz}, 3 \mathrm{H}, \mathrm{CH}_{3}\right), 0.98\left(\mathrm{~d}, J=6.4 \mathrm{~Hz}, 3 \mathrm{H}, \mathrm{CH}_{3}\right) 1.42\left(\mathrm{~s}, 9 \mathrm{H}, \mathrm{C}\left(\mathrm{CH}_{3}\right)_{3}\right), 2.32-2.56$ (septet, $J=$ $\left.6.4 \mathrm{~Hz}, 1 \mathrm{H}, \mathrm{NCH}\left(\mathrm{CH}_{3}\right)_{2}\right), 2.88-3.11$ (m, 2H, H-3, H-4), 3.35 (d, J=5.3 Hz, 1H, H-4'), 3.75 (d, J $=7.5 \mathrm{~Hz}, 1 \mathrm{H}, \mathrm{H}-2$ ), 4.18 (Appt triplet, $J=8.0 \mathrm{~Hz}, 1 \mathrm{H} \mathrm{PhOCHHCH}$ ), 4.37 (Appt triplet, $J=8.0$ $\mathrm{Hz}, 1 \mathrm{H} \mathrm{PhOCHHCH}), 6.78-7.12(\mathrm{~m}, 3 \mathrm{H}, \mathrm{Ph} H), 7.27(\mathrm{t}, J=10 \mathrm{~Hz}, 2 \mathrm{H}, \mathrm{Ph} H) .{ }^{13} \mathrm{C}$ NMR $(75$

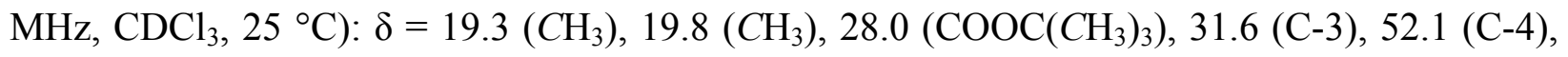

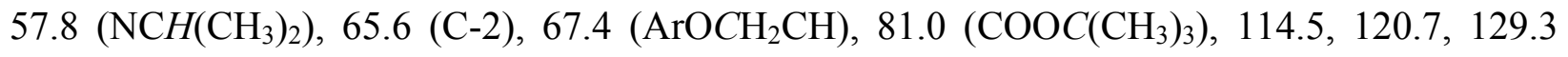
(CPh), 158.7 (ipso-CPh), 170.4 (CO) ppm. CIMS: $m / z(\%)=328$ (20), 272 (100), 250 (55), 290 (82), 204 (25). HRMS (ESI) calcd. for $\mathrm{C}_{18} \mathrm{H}_{28} \mathrm{NO}_{3}\left[\mathrm{MH}^{+}\right]$: 306.2069; found 306.2077.

(2R, 3S)-(+)-1-Isopropyl-3-phenoxymethyl-azetidine-2-carboxylic acid tert-butyl ester 45. (Table 2, Entry 10) Prepared according to GP-2. The pure compound was obtained after flash chromatography $\left(\mathrm{SiO}_{2}, \mathrm{PE} / \mathrm{AcOEt}, 4: 1\right)$ as colourless oil. Yield: $87 \mathrm{mg}(75 \%) ; R_{\mathrm{f}}=0.45$ (PE/AcOEt, 9:1); $[\alpha]_{\mathrm{D}}{ }^{20}:+48.7\left(\mathrm{c}=1.10, \mathrm{CHCl}_{3}\right) .{ }^{1} \mathrm{H} \mathrm{NMR}\left(300 \mathrm{MHz}, \mathrm{CDCl}_{3}, 25{ }^{\circ} \mathrm{C}\right): 1.26(\mathrm{~s}$, 9H, $\left.\mathrm{C}\left(\mathrm{CH}_{3}\right)_{3}\right), 2.86-3.07$ (m, 2H, H-3, H-4), 3.18 (dd, $\left.J=4.8 \mathrm{~Hz}, J=1.9 \mathrm{~Hz}, 1 \mathrm{H}, \mathrm{H}-4^{\prime}\right), 3.58$ (d, $J=12.5 \mathrm{~Hz}, 1 \mathrm{H}, \mathrm{A}$ of AB system, NCHHPh), 3.69 (d, $J=12.5 \mathrm{~Hz}, 1 \mathrm{H}, \mathrm{B}$ of AB system, $\mathrm{NCH} H \mathrm{Ph}$ ), 3.74 (d, $J=8.0 \mathrm{~Hz}, 1 \mathrm{H}, \mathrm{H}-2), 4.12$ (Appt triplet, $J=8.5 \mathrm{~Hz}, 1 \mathrm{H} \mathrm{PhOCHHCH}), 4.29$ $(\mathrm{dd}, J=9.2 \mathrm{~Hz}, J=6.5 \mathrm{~Hz}, 1 \mathrm{H} \mathrm{PhOCHHCH}), 6.82-7.04(\mathrm{~m}, 3 \mathrm{H}, \mathrm{PhH}), 7.10-7.30$ (m, 7H, $\mathrm{Ph} H)$. ${ }^{13} \mathrm{C} \mathrm{NMR}\left(75 \mathrm{MHz}, \mathrm{CDCl}_{3}, 25{ }^{\circ} \mathrm{C}\right): \delta=28.0\left(\mathrm{COOC}\left(\mathrm{CH}_{3}\right)_{3}\right), 33.3(\mathrm{C}-3), 53.1(\mathrm{C}-4), 61.9$

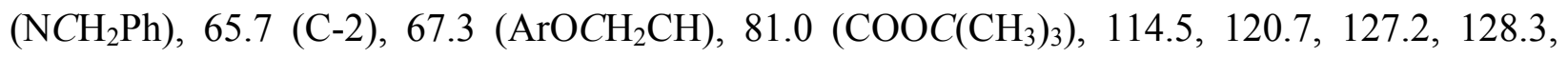
129.3, 129.4 (CPh), 137.1, 158.8 (ipso-CPh), 170.0 (CO) ppm. CIMS: $m / z(\%)=392$ (6), 376 (100), 354 (3) $\left[\mathrm{MH}^{+}\right], 298$ (72), 204 (25). HRMS (ESI) calcd. for $\mathrm{C}_{22} \mathrm{H}_{28} \mathrm{NO}_{3}\left[\mathrm{MH}^{+}\right]$: 354.2069; found 354.2071 .

$(2 R, 3 S)-(+)$ 3-Methyl-azetidine-1,2-dicarboxylic acid di-tert-butyl ester 46. To a mixture of azetidine (100 mg, $0.38 \mathrm{mmole})$, Boc anhydride ( $104 \mathrm{mg}, 0.48 \mathrm{mmol})$ in absolute ethanol (10 $\mathrm{mL}$ ) was added $\mathrm{Pd}(\mathrm{OH})_{2}$ and the reaction mixture was stirred under hydrogen ballon pressure for $2 \mathrm{~h}$. After the completion of the reaction, the reaction mixture was filtered through Celite and the filtrate was concentrated and dried under vacuo. The crude residue was then purified by silica gel column chromatography using petroleum ether and ethyl acetate (8:2) gave $N$-Boc azetidine as a 
thick oil. Yield: $92 \mathrm{mg}(89 \%) ; R_{\mathrm{f}}=0.45(\mathrm{PE} / \mathrm{AcOEt}, 4: 1) ;[\alpha]_{\mathrm{D}}{ }^{20}:+67.4\left(\mathrm{c}=0.40, \mathrm{CHCl}_{3}\right) .{ }^{1} \mathrm{H}$ NMR $\left(300 \mathrm{MHz}, \mathrm{CDCl}_{3}, 25^{\circ} \mathrm{C}\right): 0.90$ (d, $\left.J=7.3 \mathrm{~Hz}, 3 \mathrm{H}, \mathrm{CH}_{3}\right), 1.12\left(\mathrm{~s}, 9 \mathrm{H}, \mathrm{COOC}\left(\mathrm{CH}_{3}\right)_{3}\right), 1.27$ (s, 9H, NCOOC $\left(\mathrm{CH}_{3}\right)_{3}$ ), 2.70 (septet, $J=6.0 \mathrm{~Hz}, 1 \mathrm{H}, \mathrm{H}-3$ ), 3.35 (Appt triplet, $J=6.7 \mathrm{~Hz}, 1 \mathrm{H}, \mathrm{H}$ 4), 3.72 (Appt triplet, $\left.J=6.7 \mathrm{~Hz}, 1 \mathrm{H}, \mathrm{H}-4^{\prime}\right), 4.28(\mathrm{~d}, J=8.9 \mathrm{~Hz}, 1 \mathrm{H}, \mathrm{H}-2) .{ }^{13} \mathrm{C} \mathrm{NMR}(75 \mathrm{MHz}$, $\left.\mathrm{CDCl}_{3}, 25^{\circ} \mathrm{C}\right): \delta=14.3\left(\mathrm{CH}_{3}\right), 27.1\left(\mathrm{COOC}\left(\mathrm{CH}_{3}\right)_{3}\right), 27.8\left(\mathrm{NCOOC}\left(\mathrm{CH}_{3}\right)_{3}\right), 28.0(\mathrm{C}-3), 54.0(\mathrm{C}-$ 4), $65.2(\mathrm{C}-2), 79.6\left(\mathrm{COOC}\left(\mathrm{CH}_{3}\right)_{3}\right), 81.6\left(\mathrm{NCOOC}_{\left.\left(\mathrm{CH}_{3}\right)_{3}\right),} 155.4\left(\mathrm{COOC}\left(\mathrm{CH}_{3}\right)_{3}\right), 168.8\right.$ $\left(\mathrm{NCOOC}\left(\mathrm{CH}_{3}\right)_{3}\right)$ ppm. CIMS: $m / z(\%)=271(100)\left[\mathrm{M}^{+}\right], 272(16)\left[\mathrm{MH}^{+}\right] \cdot \mathrm{C}_{14} \mathrm{H}_{25} \mathrm{NO}_{4}(271.35)$ : calcd. C 61.97, H 9.29, N 5.56; found C 61.89, H 9.36, N 5.61.

(2R,3S)-(+)-3-Methyl-azetidine-2-carboxylic acid 47. To a solution of $N$-Boc azetidine 46 (92 $\mathrm{mg}, 0.34 \mathrm{mmole})$ in THF $(5 \mathrm{~mL})$ was added TFA $(3 \mathrm{~mL})$ and stirred overnight. The reaction mixture was concentrated under vacuo. The traces of TFA in the reaction mixture were removed by adding acetone and subsequent removal of the solvents from the mixture. The obtained triflate salt was purified using ion exchange Dowex resin. The column was first eluted with distilled water to get rid of trifluoroacetic acid and then the column was eluted with $\mathrm{EtOH} / \mathrm{NH}_{4} \mathrm{OH}(30 \%$ aq.soln) $/ \mathrm{H}_{2} \mathrm{O}, 9: 3: 1$ solution to elute the azetidinic amino acid. The pure compound was obtained as a colourless solid. Yield: $33 \mathrm{mg}(94 \%) ; \mathrm{Mp}: 65{ }^{\circ} \mathrm{C} ; R_{\mathrm{f}}=0.45\left(\mathrm{EtOH} / \mathrm{NH}_{4} \mathrm{OH}(30 \%\right.$ aq.soln $\left.) / \mathrm{H}_{2} \mathrm{O}, 9: 3: 1\right) ;[\alpha]_{\mathrm{D}}{ }^{20}:+210\left(\mathrm{c}=0.10, \mathrm{H}_{2} \mathrm{O}\right) .{ }^{1} \mathrm{H}$ NMR $\left(300 \mathrm{MHz}, \mathrm{D}_{2} \mathrm{O}, 25{ }^{\circ} \mathrm{C}\right): 1.07(\mathrm{~d}, J$ $\left.=7.4 \mathrm{~Hz}, 3 \mathrm{H}, \mathrm{CH}_{3}\right), 3.09$ (septet, $\left.J=7.2 \mathrm{~Hz}, 1 \mathrm{H}, \mathrm{H}-3\right), 3.48$ (dd, $J=10.4 \mathrm{~Hz}, J=6.3 \mathrm{~Hz}, 1 \mathrm{H}, \mathrm{H}-$ 4), 4.11 (Appt triplet, $J=9.8 \mathrm{~Hz}, 1 \mathrm{H}, \mathrm{H}-4), 4.75$ (d, $J=9.4 \mathrm{~Hz}, 1 \mathrm{H}, \mathrm{H}-2) .{ }^{13} \mathrm{C}$ NMR (75 MHz, $\left.\mathrm{D}_{2} \mathrm{O}, 25{ }^{\circ} \mathrm{C}\right): \delta=14.3\left(\mathrm{CH}_{3}\right), 29.3(\mathrm{C}-3), 49.9(\mathrm{C}-4), 62.9(\mathrm{C}-2), 171.7(\mathrm{CO}) \mathrm{ppm}$. CIMS: $\mathrm{m} / z$ $(\%)=133$ (20) $\left[\mathrm{MH}+\mathrm{NH}_{3}^{+}\right], 116$ (100) $\left[\mathrm{MH}^{+}\right]$. HRMS (ESI) calcd. for $\mathrm{C}_{5} \mathrm{H}_{10} \mathrm{NO}_{2}\left[\mathrm{MH}^{+}\right]$: 116.0712; found 116.0721 .

\section{Dipeptide 49}

To a solution of $N$-Benzyl azetidine 41 (219 mg, 0.84 mmole) in DCM (5 mL) was added TFA $(1 \mathrm{~mL})$ and stirred for $30 \mathrm{~min}$. The reaction mixture was concentrated under vacuo. Traces of TFA in the reaction mixture were removed by adding acetone and subsequent removal of the solvents from the mixture. To a solution of crude $N$-Benzyl azetidinic triflate salt 48, (270 mg, $0.88 \mathrm{mmol})$ in acetonitrile $(10 \mathrm{~mL})$ were added BOP reagent $(777 \mathrm{mg}, 1.76 \mathrm{mmol}), \mathrm{L}$-Ala-OMe, $\mathrm{HCl}(247 \mathrm{mg}, 1.76 \mathrm{mmol})$, and triethylamine $(0.218 \mathrm{~mL}, 1.57 \mathrm{mmol})$. After $16 \mathrm{~h}$ of stirring at r.t., the reaction was quenched by the addition of saturated aq. sodium chloride solution. The reaction mixture was extracted with ethyl acetate and the combined organic layer was washed successively with $2 \mathrm{~N} \mathrm{HCl}$, water, $5 \% \mathrm{NaHCO}_{3}$, and water and dried over $\mathrm{Na}_{2} \mathrm{SO}_{4}$. The solvent was removed under reduced pressure to give the crude dipeptide. Purification of the crude product on flash silica gel column chromatography (AcOEt/PE: 1:1) gave dipeptide 49. Yield: $166 \mathrm{mg}(65 \%) ; R_{\mathrm{f}}=0.70(\mathrm{PE} / \mathrm{AcOEt}, 9: 1) ;[\alpha]_{\mathrm{D}}{ }^{20}:+42.1\left(\mathrm{c}=1.30, \mathrm{CHCl}_{3}\right) .{ }^{1} \mathrm{H}$ NMR $(300 \mathrm{MHz}$, $\mathrm{CDCl}_{3}, 25^{\circ} \mathrm{C}$ ): $1.01\left(\mathrm{~d}, J=7.3 \mathrm{~Hz}, 3 \mathrm{H}, \mathrm{CH}_{3}\right), 1.50$ (d, $J=7.3 \mathrm{~Hz}, 3 \mathrm{H}, \mathrm{CH}_{3}$ ), 2.62-2.78 (septet, $J$ $=7.3 \mathrm{~Hz}, 1 \mathrm{H}, \mathrm{H}-3), 3.01(\mathrm{dd}, J=6.9 \mathrm{~Hz}, J=2.1 \mathrm{~Hz}, 1 \mathrm{H}, \mathrm{H}-4), 3.14$ (Appt triplet, $J=7.6 \mathrm{~Hz}, 1 \mathrm{H}$, H-4'), 3.51 (d, $J=12.3 \mathrm{~Hz}, 1 \mathrm{H}, \mathrm{A}$ of AB system, NCHHPh), $3.58(\mathrm{~d}, J=12.5 \mathrm{~Hz}, 1 \mathrm{H}, \mathrm{B}$ of AB system, $\mathrm{NCH} H \mathrm{Ph}$ ), $3.66\left(\mathrm{~s}, 3 \mathrm{H}, \mathrm{OCH}_{3}\right), 3.70(\mathrm{~d}, J=8.9 \mathrm{~Hz}, 1 \mathrm{H}, \mathrm{H}-2), 4.38$ (quintet, $J=7.5 \mathrm{~Hz}$, 
$\left.1 \mathrm{H}, \mathrm{CH}_{3} \mathrm{CHCOOCH}_{3}\right), 7.10-7.30(\mathrm{~m}, 5 \mathrm{H}, \mathrm{Ph} H), 7.40$ (br. s, $\left.1 \mathrm{H}, \mathrm{NH}\right) .{ }^{13} \mathrm{C} \mathrm{NMR}(75 \mathrm{MHz}$, $\left.\mathrm{CDCl}_{3}, 25{ }^{\circ} \mathrm{C}\right): \delta=15.2\left(\mathrm{CH}_{3}\right), 18.1\left(\mathrm{CH}_{3}\right), 27.9(\mathrm{C}-3), 46.9\left(\mathrm{CH}_{3} \mathrm{CHCOOCH}_{3}\right), 52.3\left(\mathrm{OCH}_{3}\right)$, $57.4(\mathrm{C}-4), 62.4\left(\mathrm{NCH}_{2} \mathrm{Ph}\right), 69.0$ (C-2), 127.5, 128.7, 129.0 (CPh) 137.7 (ipso-CPh), 170.6 (CO), 173.3 (CO) ppm. CIMS: $m / z(\%)=330(9), 313(100), 291(51)\left[\mathrm{MH}^{+}\right]$. HRMS (ESI) calcd. for $\mathrm{C}_{16} \mathrm{H}_{23} \mathrm{~N}_{2} \mathrm{O}_{3}\left[\mathrm{MH}^{+}\right]$: 291.1709; found 291.1694.

\section{Tripeptide 50}

To a mixture of dipeptide 49 (140 $\mathrm{mg}, 0.48 \mathrm{mmole})$ and acetic acid $(0.27 \mathrm{~mL}, 4.80 \mathrm{mmol})$ in methanol $(10 \mathrm{~mL})$ was added $\mathrm{Pd}(\mathrm{OH})_{2}(50 \mathrm{mg})$ and the reaction mixture was stirred under hydrogen ballon pressure for $2 \mathrm{~h}$. After the completion of the reaction, the reaction mixture was filterd through Celite and the filtrate was concentrated and dried under vacuo to give crude $N$ debenzylated intermediate as an ammonium acetate salt (118 $\mathrm{mg}, 95 \%)$. To a mixture of this dipeptide acetate salt $(55 \mathrm{mg}, 0.21 \mathrm{mmol})$, BOP reagent $(188 \mathrm{mg}, 0.42 \mathrm{mmol}), N$-Boc alanine $(81$ $\mathrm{mg}, 0.42 \mathrm{mmol})$ in acetonitrile $(2 \mathrm{~mL})$ was added triethylamine $(0.12 \mathrm{~mL}, 0.85 \mathrm{mmole})$. The reaction mixture was stirred at $\mathrm{rt}$ for overnight. The reaction mixture was quenched by the addition of saturated aq. sodium chloride solution and the reaction mixture was extracted with ethyl acetate $(2 \times 15 \mathrm{~mL})$. The combined organic layer were washed successively with $2 \mathrm{~N} \mathrm{HCl}$, water, $5 \% \mathrm{NaHCO}_{3}$, and water and dried with $\mathrm{Na}_{2} \mathrm{SO}_{4}$. The solvent was removed under reduced pressure to give the crude dipeptide. Purification of the crude product on flash silica gel column chromatography (AcOEt/PE: 8:2) gave tripeptide $(39 \mathrm{mg}, 50 \%) ; R_{\mathrm{f}}=0.70(\mathrm{AcOEt}) ;[\alpha]_{\mathrm{D}}{ }^{20}$ : $+85.0\left(\mathrm{c}=0.30, \mathrm{CHCl}_{3}\right) .{ }^{1} \mathrm{H}$ NMR $\left(300 \mathrm{MHz}\right.$, Acetone- $d_{6}, 25{ }^{\circ} \mathrm{C}$, mixture of rotamers $): 0.68(\mathrm{~d}$, $\left.J=6.8 \mathrm{~Hz}, 3 \mathrm{H}, \mathrm{CH}_{3}\right), 1.03\left(\mathrm{~d}, J=7.3 \mathrm{~Hz}, 3 \mathrm{H}, \mathrm{CH}_{3}\right), 1.06\left(\mathrm{~d}, J=6.8 \mathrm{~Hz}, 3 \mathrm{H}, \mathrm{CH}_{3}\right), 1.14(\mathrm{~d}, J=$ $7.0 \mathrm{~Hz}, 3 \mathrm{H}, \mathrm{CH}_{3}$ ), 1.29 (s, 9H, C(CH $\left.)_{3}\right), 2.90$ (septet, $J=8.0 \mathrm{~Hz}, 1 \mathrm{H}, \mathrm{H}-3$ ), 3.24 (septet, $J=6.6$ $\mathrm{Hz}, 1 \mathrm{H}, \mathrm{H}-3), 3.54$ (s, 3H, OCH $), 3.56\left(\mathrm{~s}, 3 \mathrm{H}, \mathrm{OCH}_{3}\right), 3.71$ (t, J=6.7 Hz, 1H, H-4), 3.76-3.96 $\left(\mathrm{m}, 2 \mathrm{H}, \mathrm{H}-4^{\prime}, \mathrm{CH}\left(\mathrm{CH}_{3}\right) \mathrm{NH}\right.$ ), 4.23 (quintet, $J=6.7 \mathrm{~Hz}, 1 \mathrm{H}, \mathrm{CH}\left(\mathrm{CH}_{3}\right) \mathrm{NH}$ ), 4.31 (quintet, $J=6.7$ $\left.\mathrm{Hz}, 1 \mathrm{H}, \mathrm{CH}\left(\mathrm{CH}_{3}\right) \mathrm{NH}\right), 4.47$ (d, $\left.J=9.4 \mathrm{~Hz}, 1 \mathrm{H}, \mathrm{H}-2\right), 5.08$ (d, $\left.J=9.0 \mathrm{~Hz}, 1 \mathrm{H}, \mathrm{H}-2\right), 5.90$ (br.s, $\mathrm{NH}), 6.25$ (br.s, NH), 7.34 (br.s, NH), 7.71 (br.s, NH). ${ }^{13} \mathrm{C}$ NMR $\left(75 \mathrm{MHz}\right.$, Acetone- $d_{6}, 25{ }^{\circ} \mathrm{C}$,

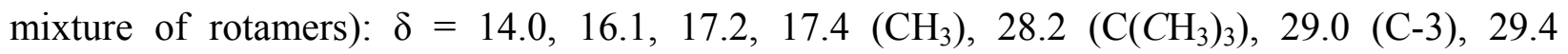
$\left(\mathrm{C}\left(\mathrm{CH}_{3}\right)_{3}\right), 46.0,47.2,47.7,47.9\left(\mathrm{NCH}\left(\mathrm{CH}_{3}\right), 52.5\left(\mathrm{OCH}_{3}\right), 54.0(\mathrm{C}-4), 55.1(\mathrm{C}-4), 64.5(\mathrm{C}-2)\right.$,

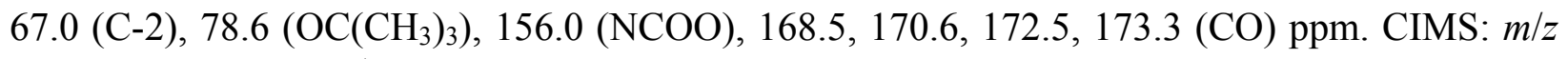
$(\%)=372(9)\left[\mathrm{MH}^{+}\right], 333$ (30), 316 (35), 260 (100), 91 (15), 82 (3). HRMS calcd. for $\mathrm{C}_{17} \mathrm{H}_{30} \mathrm{~N}_{3} \mathrm{O}_{6}\left[\mathrm{MH}^{+}\right]: 372.2135$; found 372.2132 .

(R)-methyl 3-(benzylamino)butanoate 54. To a solution of $(R)$-methyl 3-aminobutanoate (1.00 $\mathrm{g}, 9.01 \mathrm{mmol})$ in $\mathrm{MeOH}(25 \mathrm{~mL})$ was added benzaldehyde $(916 \mu \mathrm{L}, 9.01 \mathrm{mmol})$ and stirred at $\mathrm{rt}$ for $1 \mathrm{~h}$ and then sodium borohydride $(410 \mathrm{mg}, 10.8 \mathrm{mmol})$ was added portionwise at $0^{\circ} \mathrm{C}$. The reaction mixture was allowed to reach rt and stirred overnight. The excess $\mathrm{NaBH}_{4}$ was quenched by $\mathrm{NH}_{4} \mathrm{Cl}$ and then it was concentrated under vacuo. The crude residue was then extracted with diethyl ether and the organic layer was concentrated and dried. Flash column chromatographic purification of the residue $\left(\mathrm{SiO}_{2}, \mathrm{PE} / \mathrm{AcOEt}, 1: 4\right)$ gave the pure compound as colourless oil. Yield: $1.12 \mathrm{~g}(60 \%) ; R_{\mathrm{f}}=0.20(\mathrm{PE} / \mathrm{AcOEt}, 1: 1) ;[\alpha]_{578}{ }^{20}:-17.7\left(\mathrm{c}=1.25, \mathrm{CHCl}_{3}\right) .{ }^{1} \mathrm{H}$ NMR 
$\left(300 \mathrm{MHz}, \mathrm{CDCl}_{3}, 25^{\circ} \mathrm{C}\right): 1.16\left(\mathrm{~d}, J=6.4 \mathrm{~Hz}, 3 \mathrm{H}, \mathrm{CH}_{3}\right), 2.39(\mathrm{dd}, J=15.1, J=6.2 \mathrm{~Hz}, 1 \mathrm{H}$, $\left.\mathrm{CHHCOOCH}_{3}\right), 2.52\left(\mathrm{dd}, J=15.4, J=6.8 \mathrm{~Hz}, 1 \mathrm{H}, \mathrm{CH} H \mathrm{COOCH}_{3}\right), 3.15($ sextet, $J=6.4 \mathrm{~Hz}, 1 \mathrm{H}$, $\left.\mathrm{CH}_{3} \mathrm{CHNCH}_{2} \mathrm{Ph}\right), 3.67$ (s, 3H, $\left.\mathrm{OCH}_{3}\right), 3.81$ (d, $J=13.0 \mathrm{~Hz}, 1 \mathrm{H}, \mathrm{A}$ of AB system, NCHHPh), $3.88(\mathrm{~d}, J=13.0 \mathrm{~Hz}, 1 \mathrm{H}, \mathrm{B}$ of $\mathrm{AB}$ system, $\mathrm{NCH} H \mathrm{Ph}), 7.20-7.40(\mathrm{~m}, 5 \mathrm{H}, \mathrm{Ph} H) .{ }^{13} \mathrm{C}$ NMR $(75$ $\left.\mathrm{MHz}, \mathrm{CDCl}_{3}, 25^{\circ} \mathrm{C}\right): \delta=20.5\left(\mathrm{CH}_{3}\right), 41.4\left(\mathrm{CH}_{2} \mathrm{COOCH}_{3}\right), 49.7\left(\mathrm{CH}_{3} \mathrm{CH}\right), 51.2\left(\mathrm{OCH}_{3}\right), 51.6$ $\left(\mathrm{NCH}_{2} \mathrm{Ph}\right), 126.9,128.2,128.5(\mathrm{CPh}) 140.4$ (ipso-CPh), $172.8(\mathrm{CO})$ ppm. EIMS: $\mathrm{m} / z(\%)=315$ (45), 293 (100), 208 (30) [ $\left.\mathrm{MH}^{+}\right], 134$ (58). $\mathrm{C}_{12} \mathrm{H}_{17} \mathrm{NO}_{2}$ (207.27): calcd. C 69.54, H 8.27, N 6.76; found C 69.48, H 8.30, N 6.71.

(R)-3-(benzylamino)butan-1-ol 55. To a solution of 54 (946 $\mathrm{mg}, 4.57 \mathrm{mmol})$ in THF (10 mL) was added lithium aluminium hydride $(347 \mathrm{mg}, 9.14 \mathrm{mmol})$ portionwise at $0{ }^{\circ} \mathrm{C}$. After stirring for ten more minutes at this temperature, the reaction mixture was refluxed for $3 \mathrm{~h}$. After the completion of reaction, the excess $\mathrm{LAH}$ was quenched by the addition of $2 \mathrm{~N} \mathrm{NaOH}(5 \mathrm{~mL})$ and the reaction mixture was filtered off. The filtrate was concentrated and dried. The crude residue was pure enough for further reactions. Yield: $787 \mathrm{mg}(97 \%) ; R_{\mathrm{f}}=0.05$ (AcOEt/MeOH, 9:1); $[\alpha]_{\mathrm{D}}{ }^{20}:-60.0\left(\mathrm{c}=0.31, \mathrm{CHCl}_{3}\right) .{ }^{1} \mathrm{H}$ NMR $\left(300 \mathrm{MHz}, \mathrm{CDCl}_{3}, 25^{\circ} \mathrm{C}\right): 1.08(\mathrm{~d}, J=6.4 \mathrm{~Hz}, 3 \mathrm{H}$, $\left.\mathrm{CH}_{3}\right), 1.40-1.52\left(\mathrm{~m}, 1 \mathrm{H}, \mathrm{CHHCH} \mathrm{H}_{2} \mathrm{OH}\right), 1.58-1.68\left(\mathrm{~m}, \mathrm{CH} \mathrm{HCH}_{2} \mathrm{OH}\right), 2.86$ (sextet, $J=6.7 \mathrm{~Hz}, 1$ $\left.\mathrm{H}, \mathrm{CH}_{3} \mathrm{CHCH}_{2}\right), 3.60-3.78\left(\mathrm{~m}, 4 \mathrm{H}, \mathrm{NCH}_{2} \mathrm{Ph}, \mathrm{CH}_{2} \mathrm{CH}_{2} \mathrm{OH}\right), 7.10-7.30(\mathrm{~m}, 5 \mathrm{H}, \mathrm{PhH}) .{ }^{13} \mathrm{C}$ NMR $\left(75 \mathrm{MHz}, \mathrm{CDCl}_{3}, 25^{\circ} \mathrm{C}\right): \delta=20.3\left(\mathrm{CH}_{3}\right), 37.1\left(\mathrm{CH}_{3} \mathrm{CHCH}_{2}\right), 51.2\left(\mathrm{NCH}_{2} \mathrm{Ph}\right), 53.6\left(\mathrm{CH}_{3} \mathrm{CHN}\right)$, $62.2\left(\mathrm{CH}_{2} \mathrm{CH}_{2} \mathrm{OH}\right) 127.2,128.4,128.6(\mathrm{CPh}) 139.7$ (ipso-CPh) ppm. EIMS: $\mathrm{m} / z(\%)=180(100)$ $\left[\mathrm{MH}^{+}\right] . \mathrm{C}_{11} \mathrm{H}_{17} \mathrm{NO}$ (179.26): calcd. C 73.70, H 9.56, N 7.81; found C 73.72, H 9.45, N 7.76.

(R)-tert-butyl 2-(N-benzyl- $N$-(4-hydroxybutan-2-yl)amino)acetate 56. To a mixture of $(R)$-3(benzylamino)butan-1-ol 55 (187 mg, $1.06 \mathrm{mmol}$ ), sodium iodide (316mg, 2.11 mmol), sodium bicarbonate $(177 \mathrm{mg}, 2.11 \mathrm{mmol})$ in DMF $(10 \mathrm{~mL})$ was added drop wise $t$-butylbromoacetate $(310 \mu \mathrm{L}, 2.11 \mathrm{mmol})$ and the resulting suspension was stirred at $\mathrm{rt}$ overnight. After the completion of reaction, the reaction mixture was poured into a 1:1 mixture of saturated aq. $\mathrm{NH}_{4} \mathrm{Cl}$ and diethyl ether and the organic layer was concentrated and dried. The crude residue was then purified by silica gel column chromatography $\left(\mathrm{SiO}_{2}, \mathrm{PE} / \mathrm{AcOEt}, 4: 1\right)$ to give $\mathbf{5 6}$ as a colourless oil. Yield: $177 \mathrm{mg}(57 \%) ; R_{\mathrm{f}}=0.50$ (PE/AcOEt, 4:1); $[\alpha]_{\mathrm{D}}{ }^{20}:-30.0\left(\mathrm{c}=0.80, \mathrm{CHCl}_{3}\right)$. ${ }^{1} \mathrm{H}$ NMR $\left(300 \mathrm{MHz}, \mathrm{CDCl}_{3}, 25{ }^{\circ} \mathrm{C}\right): 0.90\left(\mathrm{~d}, J=6.7 \mathrm{~Hz}, 3 \mathrm{H}, \mathrm{CH}_{3}\right), 1.38-1.43(\mathrm{~m}, 1 \mathrm{H}$, $\left.\mathrm{CH}_{3} \mathrm{CHCHH}\right), 1.38\left(\mathrm{~s}, 9 \mathrm{H}, \mathrm{C}\left(\mathrm{CH}_{3}\right)_{3}\right), 1.58-1.73\left(\mathrm{~m}, 1 \mathrm{H} \mathrm{CH}_{3} \mathrm{CHCH} H\right), 2.81-2.95(\mathrm{~m}, 1 \mathrm{H}$, $\left.\mathrm{CH}_{3} \mathrm{CHN}\right), 2.96\left(\mathrm{~d}, J=16.8 \mathrm{~Hz}, \mathrm{NCHHCOO}^{t} \mathrm{Bu}\right), 3.26\left(\mathrm{~d}, J=16.8 \mathrm{~Hz}, \mathrm{NCH} H \mathrm{COO}{ }^{t} \mathrm{Bu}\right), 3.30$ $(\mathrm{d}, J=13.3 \mathrm{~Hz}, 1 \mathrm{H}, \mathrm{A}$ of $\mathrm{AB}$ system, $\mathrm{NCHHPh}), 3.47(\mathrm{dt}, J=9.7 \mathrm{~Hz}, J=3.6 \mathrm{~Hz}, 1 \mathrm{H}$, $\left.\mathrm{CH}_{2} \mathrm{CHHOH}\right), 3.65-3.77$ (m, $\left.2 \mathrm{H}, \mathrm{CH}_{2} \mathrm{CH} H \mathrm{OH}, \mathrm{NCH} H \mathrm{Ph}\right), 7.10-7.30(\mathrm{~m}, 5 \mathrm{H}, \mathrm{Ph} H) .{ }^{13} \mathrm{C}$ NMR $\left(75 \mathrm{MHz}, \mathrm{CDCl}_{3}, 25^{\circ} \mathrm{C}\right): \delta=12.6\left(\mathrm{CH}_{3}\right), 28.1\left(\mathrm{C}\left(\mathrm{CH}_{3}\right)_{3}\right), 35.6\left(\mathrm{CH}_{3} \mathrm{CHCH}_{2}\right), 51.9\left(\mathrm{NCH}_{2} \mathrm{Ph}\right)$, $54.3\left(\mathrm{NCH}_{2} \mathrm{COO}^{\mathrm{t}} \mathrm{Bu}\right), 54.4\left(\mathrm{CH}_{3} \mathrm{CHN}\right), 62.0\left(\mathrm{CH}_{2} \mathrm{CH}_{2} \mathrm{OH}\right), 81.7\left(C\left(\mathrm{CH}_{3}\right)_{3}\right), 127.3,128.5,129.0$

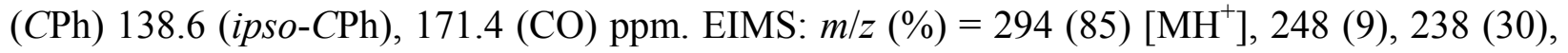
220 (9), 193 (17), 192 (100), 148 (13), 91 (30). $\mathrm{C}_{17} \mathrm{H}_{27} \mathrm{NO}_{3}$ (179.26): calcd. C 69.59, H 9.28, N 4.77; found C 69.62, H 9.30, N 4.74.

(1R)-(-)-1-Benzyl-(3-chloro-1-methyl-propyl)-amino]-acetic acid tert-butyl ester 57. Prepared according to GP-1. The pure compound was obtained after flash chromatography $\left(\mathrm{SiO}_{2}\right.$, 
PE/AcOEt, 9:1) as a colourless oil. Yield: $600 \mathrm{mg}(78 \%) ; R_{\mathrm{f}}=0.70(\mathrm{PE} / \mathrm{AcOEt}, 9: 1) ;[\alpha]_{\mathrm{D}}{ }^{20}:-$ $39.1\left(\mathrm{c}=0.70, \mathrm{CHCl}_{3}\right) .{ }^{1} \mathrm{H} \mathrm{NMR}\left(300 \mathrm{MHz}, \mathrm{CDCl}_{3}, 25{ }^{\circ} \mathrm{C}\right): 0.95\left(\mathrm{~d}, J=6.6 \mathrm{~Hz}, 3 \mathrm{H}, \mathrm{CH}_{3}\right), 1.36$ $\left(\mathrm{s}, 9 \mathrm{H}, \mathrm{C}\left(\mathrm{CH}_{3}\right)_{3}\right), 1.54-1.68\left(\mathrm{~m}, 1 \mathrm{H}, \mathrm{ClCH}_{2} \mathrm{CHH}\right), 1.82-1.96\left(\mathrm{~m}, 1 \mathrm{H}, \mathrm{ClCH}_{2} \mathrm{CH} H\right), 2.83-2.96(\mathrm{~m}$,

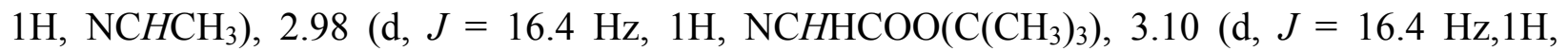
$\mathrm{NCH} H C O O\left(\mathrm{C}\left(\mathrm{CH}_{3}\right)_{3}\right) 3.51(\mathrm{~d}, J=13.8 \mathrm{~Hz}, 1 \mathrm{H}, \mathrm{A}$ of AB system, $\mathrm{NCHHPh}), 3.92(\mathrm{~d}, J=14.1$ $\mathrm{Hz}, 1 \mathrm{H}, \mathrm{B}$ of $\mathrm{AB}$ system, $\mathrm{NCH} H \mathrm{Ph}), 3.56-3.67\left(\mathrm{~m}, 2 \mathrm{H}, \mathrm{CH}_{2} \mathrm{Cl}\right), 3.73(\mathrm{~d}, J=13.8 \mathrm{~Hz}, 1 \mathrm{H}, \mathrm{B}$ of $\mathrm{AB}$ system, NCHHPh), 7.10-7.34 (m, 5H, $\mathrm{Ph} H) .{ }^{13} \mathrm{C} \mathrm{NMR}\left(75 \mathrm{MHz}, \mathrm{CDCl}_{3}, 25{ }^{\circ} \mathrm{C}\right): \delta=14.0$ $\left(\mathrm{CH}_{3}\right), 28.1\left(\mathrm{COOC}\left(\mathrm{CH}_{3}\right)_{3}\right), 37.8\left(\mathrm{ClCH}_{2} \mathrm{CH}_{2}\right), 42.8\left(\mathrm{ClCH}_{2}\right), 52.2\left(\mathrm{NCH}_{2} \mathrm{COOC}\left(\mathrm{CH}_{3}\right)_{3}\right), 53.0$

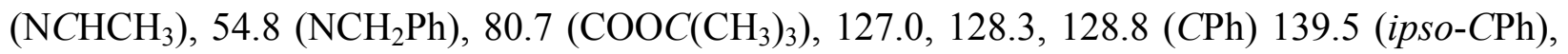
171.5 (CO) ppm. CIMS: m/z (\%) = 346 (14), 312 (100) $\left[\mathrm{MH}^{+}\right], 311$ (40), 256 (17), 210 (93). $\mathrm{C}_{17} \mathrm{H}_{26} \mathrm{ClNO} 2$ (311.85): calcd. C 65.48, H 8.40, N 4.49; found C 65.40, H 8.42, N 4.53.

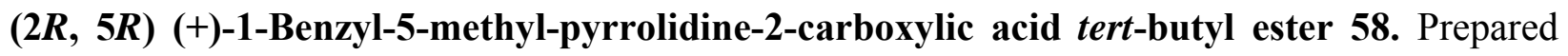
according to GP-2. The pure compound was obtained after flash chromatography $\left(\mathrm{SiO}_{2}\right.$, PE/AcOEt, 9:1) as a colourless oil. Yield: $82 \mathrm{mg}(59 \%) ; R_{\mathrm{f}}=0.57$ (PE/AcOEt, 9:1); $[\alpha]_{\mathrm{D}}{ }^{20}:+$ $13.5\left(\mathrm{c}=0.31, \mathrm{CHCl}_{3}\right) .{ }^{1} \mathrm{H} \mathrm{NMR}\left(300 \mathrm{MHz} \mathrm{CDCl}_{3}, 25^{\circ} \mathrm{C}\right): 1.15\left(\mathrm{~d}, J=6.0 \mathrm{~Hz}, 3 \mathrm{H}, \mathrm{CH}_{3}\right), 1.40$ (s, 9H, C( $\left.\left.\mathrm{CH}_{3}\right)_{3}\right), 1.50-1.68$ (m,1H, H-4), 1.80-2.03 (m, 3H, H-3, H-3', H-4'), 2.68-2.82 (m, 1H, H-5), 3.21 (Appt triplet, $J=7.0 \mathrm{~Hz}, 1 \mathrm{H}, \mathrm{H}-2), 3.79$ (d, $J=14.1 \mathrm{~Hz}, 1 \mathrm{H}$, A of AB system, $\mathrm{NCHHPh}), 3.92(\mathrm{~d}, J=14.1 \mathrm{~Hz}, 1 \mathrm{H}, \mathrm{B}$ of AB system, $\mathrm{NCH} H \mathrm{Ph}), 7.22-7.40(\mathrm{~m}, 5 \mathrm{H}, \mathrm{Ph} H) .{ }^{13} \mathrm{C}$

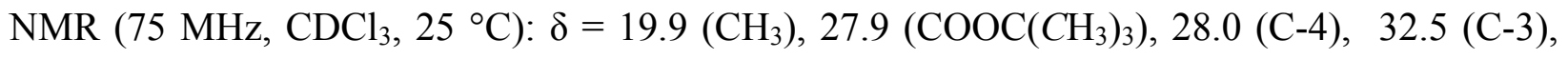

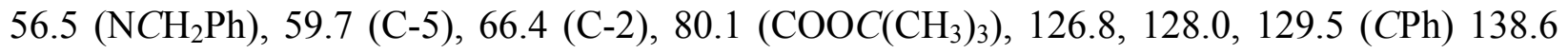
(ipso-CPh), 173.9 (CO) ppm. CIMS: $m / z(\%)=276(42)\left[\mathrm{MH}^{+}\right], 220$ (26), 174 (100), 91 (34). $\mathrm{C}_{17} \mathrm{H}_{25} \mathrm{NO}_{2}$ (275.39): calcd. C 74.14, H 9.15, N 5.09; found C 74.10, H 9.09, N 5.17.

(2S, 5R)-(-)-1-Benzyl-5-methyl-pyrrolidine-2-carboxylic acid tert-butyl ester 59. Prepared according to GP-2. The pure compound was obtained after flash chromatography $\left(\mathrm{SiO}_{2}\right.$, PE/AcOEt, 9:1) as a colourless oil. Yield: $54 \mathrm{mg}(39 \%) ; R_{\mathrm{f}}=0.60(\mathrm{PE} / \mathrm{AcOEt}, 9: 1) ;[\alpha]_{\mathrm{D}}{ }^{20}:-$ $81.6\left(\mathrm{c}=0.75, \mathrm{CHCl}_{3}\right) .{ }^{1} \mathrm{H} \mathrm{NMR}\left(300 \mathrm{MHz}, \mathrm{CDCl}_{3}, 25^{\circ} \mathrm{C}\right): 1.11\left(\mathrm{~d}, J=6.2 \mathrm{~Hz}, 3 \mathrm{H}, \mathrm{CH}_{3}\right), 1.49$ (s, $\left.10 \mathrm{H}, \mathrm{H}-4, \mathrm{C}\left(\mathrm{CH}_{3}\right)_{3}\right), 1.70-1.82(\mathrm{~m}, 1 \mathrm{H}, \mathrm{H}-4)$ ), 2.05-2.28 (m, 2H, H-3, H-3'), 3.31-3.45 (sextet, $J=6.8 \mathrm{~Hz}, 1 \mathrm{H}, \mathrm{H}-5), 3.50(\mathrm{dd}, J=5.8 \mathrm{~Hz}, J=2.5 \mathrm{~Hz}, 1 \mathrm{H}, \mathrm{H}-2), 3.76(\mathrm{~d}, J=13.5 \mathrm{~Hz}, 1 \mathrm{H}$, A of AB system, NCHHPh), 3.98 (d, $J=13.7 \mathrm{~Hz}, 1 \mathrm{H}, \mathrm{B}$ of AB system, NCHHPh ), 7.11-7.42 (m, 5H, $\mathrm{PhH}) .{ }^{13} \mathrm{C}$ NMR $\left(75 \mathrm{MHz}, \mathrm{CDCl}_{3}, 25{ }^{\circ} \mathrm{C}\right): \delta=19.7\left(\mathrm{CH}_{3}\right), 27.4(\mathrm{C}-4), 28.2\left(\mathrm{COOC}\left(\mathrm{CH}_{3}\right)_{3}\right), 31.8$

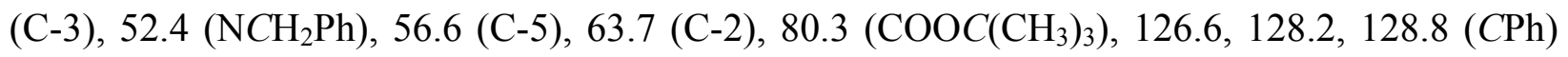
140.2 (ipso-CPh), 174.2 (CO) ppm. CIMS: $m / z(\%)=276(29)\left[\mathrm{MH}^{+}\right], 220$ (13), 174 (100), 91 (23). $\mathrm{C}_{17} \mathrm{H}_{25} \mathrm{NO}_{2}$ (275.39): calcd. C 74.14, H 9.15, N 5.09; found C 74.20, H 9.19, N 5.02.

[Benzyl-(3-chloro-butyl)-amino]-acetic acid tert-butyl ester 62. Prepared according to GP-1. The pure compound was obtained after flash chromatography $\left(\mathrm{SiO}_{2}, \mathrm{PE} / \mathrm{AcOEt}\right.$, 9:1) as a colourless oil. Yield: $1.77 \mathrm{~g}(93 \%) ; R_{\mathrm{f}}=0.70$ (PE/AcOEt, 9:1); ${ }^{1} \mathrm{H}$ NMR (300 MHz, $\mathrm{CDCl}_{3}, 25$ $\left.{ }^{\circ} \mathrm{C}\right): 1.54\left(\mathrm{~s}, 12 \mathrm{H}, \mathrm{C}\left(\mathrm{CH}_{3}\right)_{3}\right), 1.92$ (quartet, $\left.J=6.7 \mathrm{~Hz}, 2 \mathrm{H}, \mathrm{NCH}_{2} \mathrm{CH}_{2} \mathrm{CHCl}\right), 2.80-2.97$ (m, $2 \mathrm{H}$, $\left.\mathrm{NCH}_{2} \mathrm{CH}_{2}\right), 3.27$ (s, $\left.2 \mathrm{H}, \mathrm{NCH}_{2} \mathrm{COOC}\left(\mathrm{CH}_{3}\right)_{3}\right), 3.85\left(\mathrm{~s}, 2 \mathrm{H}, \mathrm{NCH}_{2} \mathrm{Ph}\right), 4.24$ (sextet, $J=6.6 \mathrm{~Hz}, 1$ $\left.\mathrm{H}, \mathrm{CH}_{3} \mathrm{CHCl}\right), 7.25-7.45(\mathrm{~m}, 5 \mathrm{H}, \mathrm{PhH}) .{ }^{13} \mathrm{C} \mathrm{NMR}\left(75 \mathrm{MHz}, \mathrm{CDCl}_{3}, 25{ }^{\circ} \mathrm{C}\right): \delta=25.5\left(\mathrm{CH}_{3}\right)$, $28.3\left(\mathrm{COOC}\left(\mathrm{CH}_{3}\right)_{3}\right), 38.6\left(\mathrm{NCH}_{2} \mathrm{CH}_{2}\right), 51.3\left(\mathrm{NCH}_{2} \mathrm{CH}_{2}\right), 55.4\left(\mathrm{NCH}_{2} \mathrm{COOC}\left(\mathrm{CH}_{3}\right)_{3}\right), 56.6$ 


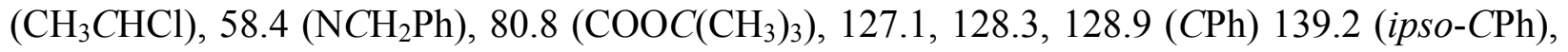
170.7 (CO) ppm. EIMS: $m / z(\%)=334$ (35) $\left[\mathrm{M}+\mathrm{Na}^{+}\right], 280$ (32), 242 (24), 200 (17). $\mathrm{C}_{16} \mathrm{H}_{24} \mathrm{ClNO}_{2}$ (297.82): calcd. C, 64.53; H, 8.12; N, 4.70; found C 64.45, H 8.06, N 4.79.

cis and trans-1-Benzyl-3-methyl-pyrrolidine-2-carboxylic acid tert-butyl ester 63. Prepared according to GP-2. The pure compound was obtained after flash chromatography $\left(\mathrm{SiO}_{2}\right.$, PE/AcOEt, 9:1) as a colourless oil. Yield: $54 \mathrm{mg}$ (98\%); $R_{\mathrm{f}}=0.60$ (PE/AcOEt, 9:1); ${ }^{1} \mathrm{H}$ NMR (300 MHz, $\mathrm{CDCl}_{3}, 25^{\circ} \mathrm{C}, 2: 1$ mixture of diasteroisomers): 0.94 (d, $J=6.9 \mathrm{~Hz}, 3 \mathrm{H}, \mathrm{CH}_{3}$ major isomer), $1.03\left(\mathrm{~d}, J=6.7 \mathrm{~Hz}, 3 \mathrm{H}, \mathrm{CH}_{3}\right.$, minor isomer), 1.11-1.41 (m, 1H, H-4, major isomer), $1.35\left(\mathrm{~s}, 9 \mathrm{H}, \mathrm{C}\left(\mathrm{CH}_{3}\right)_{3}\right.$, minor isomer), $1.38\left(\mathrm{~s}, 9 \mathrm{H}, \mathrm{C}\left(\mathrm{CH}_{3}\right)_{3}\right.$, major isomer), $1.42-1.60(\mathrm{~m}, 1 \mathrm{H}, \mathrm{H}-$ 4, major isomer), 1.83-2.07 (m, 1H, H-4') , 2.18-2.45 (m, 2H, H-3, H-5, major isomer), 2.60 (d, J $=7.3 \mathrm{~Hz}, 1 \mathrm{H}, \mathrm{H}-2$, major isomer), 2.85-3.00 (m, $1 \mathrm{H}, \mathrm{H}-5), 3.19(\mathrm{~d}, J=8.3 \mathrm{~Hz} 1 \mathrm{H}, \mathrm{H}-2$, minor isomer), 3.33 (d, $J=12.3 \mathrm{~Hz}, 1 \mathrm{H}, \mathrm{A}$ of $\mathrm{AB}$ system, $\mathrm{NCHHPh}$, major isomer), 3.56 (d, $J=13.1$ $\mathrm{Hz}, 1 \mathrm{H}, \mathrm{A}$ of $\mathrm{AB}$ system, $\mathrm{NCHHPh}$, minor isomer), 3.75 (d, $J=12.9 \mathrm{~Hz}, 1 \mathrm{H}, \mathrm{B}$ of AB system, $\mathrm{NCH} H \mathrm{Ph}$, minor isomer) $3.85(\mathrm{~d}, J=12.9 \mathrm{~Hz}, 1 \mathrm{H}, \mathrm{B}$ of $\mathrm{AB}$ system, $\mathrm{NCH} H \mathrm{Ph}$, minor isomer), 7.10-7.38 (m, 5H, $\mathrm{PhH}) .{ }^{13} \mathrm{C} \mathrm{NMR}\left(75 \mathrm{MHz}, \mathrm{CDCl}_{3}, 25{ }^{\circ} \mathrm{C}\right.$, major diasteromer): $\delta=20.0\left(\mathrm{CH}_{3}\right)$, $28.2\left(\mathrm{COOC}\left(\mathrm{CH}_{3}\right)_{3}\right), 31.3(\mathrm{C}-4), 36.1(\mathrm{C}-3), 51.8(\mathrm{C}-5), 58.7\left(\mathrm{NCH}_{2} \mathrm{Ph}\right), 74.1(\mathrm{C}-2), 80.5$ $\left(\mathrm{COOC}\left(\mathrm{CH}_{3}\right)_{3}\right), 126.9,128.7,129.2(\mathrm{CPh}) 138.7$ (ipso-CPh), $172.9(\mathrm{CO}) \mathrm{ppm}$. CIMS: $\mathrm{m} / z(\%)=$ $276(35)\left[\mathrm{MH}^{+}\right], 221$ (10), 173 (100), 91 (20). $\mathrm{C}_{17} \mathrm{H}_{25} \mathrm{NO}_{2}$ (275.39): calcd. C 74.14, H 9.15, N 5.09; found C 74.10, H 9.17, N 5.13.

\section{References}

1. (a) Hart, P. A.; Rich, D. H.; In The Practice of Medicinal Chemistry, Wermuth, C. G. Ed. Academic Press, $3^{\text {rd }}$ Edn., 2000. (b) Hanessian, S.; MacNaughton-Smith, G.; Lombart, H. G.; Lubell, W. D. Tetrahedron 1997, 53, 12789. (c) Giannis, A.; Kolter, T. Angew. Chem., Int. Ed. 1993, 32, 1244.

2. Lorthiois, E.; Marek, I.; Normant, J-F. Tetrahedron Lett. 1997, 38, 89. For recent examples, see: (a) Karoyan, P.; Quancard, J.; Vaissermann, J.; Chassaing, G. J. Org. Chem. 2003, 68, 2256.(b) Quancard, J.; Labonne, A.; Jacquot, Y.; Chassaing G.; Lavielle S.; Karoyan P. J. Org. Chem. 2004, 69, 7940.

3. (a) Couty, F. Amino Acids 1999, 16, 397. (b) Kadouri-Puchot, C.; Comesse, S. Amino Acids 2005, 29, 101.

4. Fowden, L. Nature 1955, 176, 347.

5. Couty, F.; Evano, G. Org. Prep. Int. Proc. 2006, in press.

6. (a) Zagari, A.; Nemethy, G.; Sheraga, H. A. Biopolymers 1990, 30, 951. (b) Zagari, A. Nemethy, G.; Sheraga, H. A. Biopolymers 1990, 30, 967. (c) Zagari, A.; Palmer, K. A.; Gibson, K. D.; Nemethy, G.; Sheraga, H. A. Biopolymers 1994, 34, 51. (d) Deming, T. J.; Fournier, M. J., Mason T. L.; Tirell, D. A. Macomolecules 1996, 29, 1442. (e) Tsai, F. H.; Overberger, C. G.; Zand R. Biopolymers 1990, 30, 1039. 
7. (a) Kozikowski, A. P.; Tückmantel, W.; Reynolds, I. J.; Wrobleski, J. T. J. Med. Chem. 1990, 33, 1561. (b) Hanessian, S.; Fu, M.J.; Chiara, J-L;. Di Fabio, R. Tetrahedron Lett. 1993, 34, 4157. (c) Hanessian, S.; Bernstein, N.;. Yang, R-Y.; Maguire, R. Bioorg. Med. Chem. Lett. 1999, 9, 1437. (d) Couty, F.; Evano, G.; Rabasso, N.; Tetrahedron: Asymmetry 2003, 14, 2407. (e) Jiang, J.; Shah, H.; DeVita, R. J. Org. Lett. 2003, 5, 4101. (f) GeronaNavarro, G.; Angeles Bonache, M.; Alías, M.; Pérez de Vega, M. J.; García-López, T.; Pilar López, M.; Cativiela, C.; González-Muñiz, R. Tetrahedron Lett. 2004, 45, 2193. (f) Sajjadi, Z.; Lubell, W. D. J. Pept. Res. 2005, 65, 298. (g) Braüner-Osborne, H.; Bunch, L.; Chopin, N.; Couty, F.; Evano, G.; Jensen, A. A.; Kusk, M.; Nielsen, B.; Rabasso, N. Org. Biomol. Chem., 2005, 3, 3926. (h) Enders, D.; Gries J. Synthesis 2005, 3508.

8. Sivaprakasam, M.; Couty, F.; Evano, G.; Srinivas, B.; Sridhar, R.; Rama Rao, K. Synlett 2006, 781.

9. Agami, C.; Couty, F.; Evano, G. Tetrahedron: Asymmetry 2002, 13, 297.

10. For recent exemples, see: (a) Couty, F.; David, O.; Durrat, F.; Evano, G.; Lakhdar, S.; Marrot, J.; Vargas-Sanchez, M. Eur. J. Org. Chem. 2006, 3479. (b) Couty, F.; Durrat, F.; Evano, G.; Marrot, J. Eur. J. Org. Chem. 2006, early view, and references cited therein.

11. Čaplar, V.; Raza, Z.; Katalenić, D.; Žinić, M. Croatica Chemica Acta 2003, 76, 23.

12. (2S,3R)-O-benzyl threoninol was prepared from $(2 S, 3 R) N$-Boc- $O$-benzyl threonine ${ }^{13}$ throug a two-step sequence involving $N$-Boc deprotection (TFA in DCM, rt, 0.5h, 95\%) followed by $\mathrm{LAH}$ reduction $\left(\mathrm{LiAlH}_{4}\right.$, THF, reflux, 3h, 36\%).

13. Yutaka, N.; Miki, H.; Kenji, T.; Yashusika, Y.; Chung-gi, S. Bull. Chem. Soc. Jpn 1995, 68, 1369.

14. Rajender Reddy, L.; Bhanumati, N.; Rama Rao, K. Chem. Commun. 2000, 2321.

15. Concellón, J. M.; Pablo, L. B.; Pérez-Andrés, J. A. Tetrahedron Lett. 2000, 41, 1231.

16. Pirkle, W. H.; Hoekstra, M. S. J. Am. Chem. Soc. 1976, 98, 1832.

17. Davies, S. G.; Smith, A. D.; Price, P. D. Tetrahedron: Asymmetry 2005, 16, 2833.

18. Shiosaki, K.; Rapoport, H. J. Org. Chem. 1985, 50, 1229. The signal of the H-2 proton appears more deshielded in the trans isomer (3.4-3.5 ppm) than in the cis isomer (3.15-3.20 ppm). 